\title{
Gut-bladder axis syndrome associated with recurrent UTIs in humans
}

Colin J. Worby ${ }^{1}$, Henry L. Schreiber IV ${ }^{2,3,4}$, Timothy J. Straub ${ }^{1}$, Lucas R. van Dijk ${ }^{1,5}$, Ryan A. Bronson ${ }^{1}$, Benjamin Olson ${ }^{2,3}$, Jerome S. Pinkner², Chloe L. P. Obernuefemann ${ }^{2}$, Vanessa L. Muñoz ${ }^{2}$, Alexandra E. Paharik ${ }^{2}$, Bruce J. Walker ${ }^{6}$, Christopher A. Desjardins ${ }^{1}$, Wen-Chi Chou ${ }^{1}$, Karla Bergeron ${ }^{7}$, Sinéad B. Chapman ${ }^{1}$, Aleksandra Klim ${ }^{7}$, Abigail L. Manson ${ }^{1}$, Thomas J. Hannan $^{8}$, Thomas M. Hooton ${ }^{9}$, Andrew L. Kau ${ }^{3,10}$, H. Henry Lai ${ }^{7,11}$, Karen W. Dodson ${ }^{2,3}$, Scott J. Hultgren $^{\star 2,3}$, Ashlee M. Earl ${ }^{\star 1}$

1. Broad Institute, Cambridge, MA 02142, USA

2. Department of Molecular Microbiology, Washington University School of Medicine, St. Louis, MO 63110, USA

3. Center for Women's Infectious Disease Research (CWIDR), Washington University School of Medicine, St. Louis, MO 63110, USA

4. Division of Biology \& Biological Engineering, California Institute of Technology, Pasadena, CA, USA

5. Delft Bioinformatics Lab, Delft University of Technology, Van Mourik Broekmanweg 6, 2628 XE, Netherlands

6. Applied Invention, Cambridge MA, USA

7. Department of Surgery, Division of Urologic Surgery, Washington University School of Medicine, St. Louis, MO 63110, USA

8. Department of Pathology and Immunology, Washington University School of Medicine, St. Louis, MO 63110, USA

9. Department of Medicine, University of Miami, Miami, Florida, USA

10. Department of Medicine, Division of Allergy and Immunology. Washington University School of Medicine, St. Louis, MO 63110, USA

11. Department of Anesthesiology, Washington University School of Medicine, St. Louis, MO 63110, USA

*To whom correspondence may be addressed (aearl@broadinstitute.org; hultgren@wustl.edu)

\section{Abstract}

Recurrent urinary tract infections (rUTIs) are a major health burden worldwide, with history of infection being a significant risk factor. While the gut is a known reservoir for uropathogenic bacteria, the role of the microbiota in rUTI remains unclear. We conducted a year-long study of women with and without history of rUTIs, from whom we collected urine, blood and monthly fecal samples for multi-omic interrogation. The rUTI gut microbiome was significantly depleted in microbial richness and butyrate-producing bacteria compared to controls, reminiscent of other inflammatory conditions, though Escherichia coli gut and bladder dynamics were comparable between cohorts. Blood samples revealed signals of differential systemic immunity, leading us to hypothesize that rUTI susceptibility is in part mediated through a syndrome involving the gut- 
bladder axis, comprising gut dysbiosis and differential immune response to bacterial bladder colonization, manifesting in symptoms. This work highlights the potential for microbiome therapeutics to prevent and treat rUTIs.

\section{Introduction}

Urinary tract infections (UTIs) are one of the most common bacterial infections worldwide and are a significant cause of morbidity in otherwise healthy females, with uropathogenic Escherichia coli (UPEC) being the primary causative agent ${ }^{1}$. One of the strongest risk factors for UTI is a history of prior UTIs ${ }^{2}$, but the biological basis for this phenomenon, and the risk factors for long-term recurrence remain unclear in otherwise healthy women. $20-30 \%$ of women diagnosed with a UTI will experience a recurrent UTI (rUTI) in the following months, with some suffering six or more per year, but the reason for this is mostly unknown. Over one million women in the United States are referred to urologists each year because of rUTIs, and the rapid spread of antibiotic resistance in uropathogens is making treatment even more challenging. The gut is a reservoir for UPEC and UTIs most commonly arise via the ascension of UPEC from the gut to the urinary tract ${ }^{3-5}$. Recent studies have explored the 'gut microbiota-UTI axis', showing that uropathogen abundance in the gut is a risk factor for UTI in kidney transplant patients ${ }^{6}$, and that a 'bloom' in uropathogen gut abundance may precede infection ${ }^{7}$. Other studies have demonstrated differences in gut microbiome composition associated with children suffering UTIs ${ }^{8}$, and with kidney transplant patients developing bacteriuria ${ }^{9}$, compared to healthy controls. Furthermore, fecal microbiota transplants to treat Clostridium difficile infections may have the collateral effect of reducing the frequency of rUTI ${ }^{10,11}$, suggesting that perturbation of the gut microbiota can modulate rUTI susceptibility.

It is increasingly accepted that the gut microbiota can play a role in conditions affecting distal organs - for instance, the gut-brain axis and the gut-lung axis are the subject of much ongoing research ${ }^{12-15}$. However, the gut-bladder axis - the spectrum of direct and indirect interactions between gut flora and the bladder immune and/or infection status - remains uncharacterized, and the role of the gut microbiota in rUTI susceptibility is not well understood. In particular, no study has yet been able to ascertain whether: i) gut dysbiosis is associated with rUTI susceptibility; ii) rUTI women have unique uropathogen dynamics within and between the gut and the bladder; or iii) microbiome-mediated immunological differences may be linked to rUTI susceptibility, as seen in a range of other diseases ${ }^{16}$.

Here, we present results from the UTI microbiome (UMB) project, a year-long clinical study of women with a history of rUTI and a matched cohort of healthy women. Our unique longitudinal study design allowed us to explore the importance and interdependence of the gut microbiota and $E$. coli strain dynamics in rUTI, susceptibility to infection, and host immune responses that may impact these dynamics. Using metagenomic techniques, including newly developed algorithms to track low abundance $E$. coli at the strain-level, we determined that: i) compared to healthy controls, women with a history of rUTI had a distinct, less diverse gut microbiota, depleted in butyrate producers and exhibiting characteristics of low-level inflammation; ii) differential immunological biomarkers suggest rUTI women may have a distinct immune state, 
iii) E. coli strains were transmitted from the gut to the bladder in both cohorts, though no UTI symptoms occurred in healthy controls, and iv) UTI-causing E. coli strains often persistently colonized the gut, and were not permanently cleared by repeated antibiotic exposure. Thus, susceptibility to rUTI is in part mediated through a syndrome involving the gut-bladder axis, comprising a dysbiotic gut microbiome with reduced butyrate production capacity and apparent alterations of systemic immunity. Our work shows that UPEC strains persist in the gut despite antibiotic treatment, which itself may exacerbate gut dysbiosis. Therapies targeting UPEC in the gut and/or the microbiome, e.g., therapeutics to attenuate gut dysbiosis, could be promising alternatives to antibiotics.

\section{Results \& Discussion}

\section{Frequent antibiotic exposure and $E$. coli infections in rUTI cohort}

Women with a history of rUTI were recruited to the UMB study, along with an age- and community-matched control cohort comprising healthy women (Methods). A total of 16 control and 15 rUTI women participated in the year-long study, providing monthly home-collected stool samples, as well as blood, urine and rectal swabs at enrollment and subsequent clinic visits for UTI treatment (Figure 1a). Participants completed monthly questionnaires on diet, symptoms, and behavior (Supp Data). There was a greater proportion of white women in the rUTI cohort, and self-reported antibiotic use was higher in this group in line with UTI treatment; otherwise, few dietary or behavioral differences were apparent (Table S1).

A total of 24 UTIs occurred during the study, all occurring in rUTI women, who each experienced between 0-4 UTIs (Figure 1b). Nineteen UTIs were diagnosed by clinicians at the study clinic, and five were inferred through self-reported UTI symptoms (painful urination, increased urgency/frequency of urination, cloudy urine) together with subsequent antibiotic use as reported in the questionnaire administered during monthly sample collection. UTIs were typically treated with ciprofloxacin or nitrofurantoin. No significant temporal risk factors for UTI were identified amongst dietary or behavioral variables. Sexual intercourse is a well-known risk factor for UTI ${ }^{2,17}$, and all 19 clinically diagnosed UTIs occurred following at least one reported sexual encounter in the previous two weeks (Figure S1).

Urine samples collected at the time of clinical UTI diagnoses were plated on MacConkey agar; bacterial growth was detected (>0 CFU/ml) from the majority $(15 / 19 ; 79 \%$, Table S2). To determine the cause of infection, we sequenced 13 urine cultures as well as uncultured urine from all UTI diagnoses, defaulting to results from cultures, when available. E. coli dominated $12 / 13(92 \%)$ sequenced outgrowths; the remaining sample was dominated by Klebsiella pneumoniae. Sequencing uncultured urine from the remaining UTI samples identified uropathogens in a further four samples, including E. coli (2), Enterococcus faecalis and Staphylococcus saprophyticus, while two yielded no bacterial sequence (Table S2). Based on sequencing, we defined 14 E. coli UTIs, comprising $82 \%$ of infections for which a bacterial 
cause could be inferred, broadly reflecting previous estimates of the proportion of all UTIs caused by E. coli ${ }^{1}$.

\section{rUTI gut is depleted in microbial richness and butyrate-producing taxa reminiscent of antibiotic exposure}

It is increasingly recognized that the gut microbiota plays a role in a range of autoimmune and inflammatory disease ${ }^{18}$, as well as susceptibility to infection ${ }^{16}$, and can alter inflammation in distal organs ${ }^{19}$. While previous studies have highlighted differential abundances of nonuropathogenic gut taxa as risk factors for bacteriuria in kidney transplant patients (reduced Faecalibacterium and Romboutsia ${ }^{9}$ ) and UTIs in children (reduced Peptostreptococcaceae ${ }^{8}$ ), it is unclear if these are also risk factors for recurrence in otherwise healthy adult women. To explore this question, we sequenced and analyzed the metagenomes of 367 longitudinal stool samples from both rUTI $(n=197)$ and control $(n=170)$ women (Figure 1b; Methods).

After adjusting for potential confounding factors (race and recent antibiotic use), we found broad differences in the composition of the gut microbiota between cohorts (Figure 2a-c). Gut microbial richness was significantly lower, on average, in rUTI women compared to controls $(p=0.05$, Figure $2 c)$. At the phylum level, we saw elevated levels of Bacteroidetes (false discovery rate $[F D R]=0.003)$ and a lower relative abundance of Firmicutes $(F D R=0.02)$ ) in rUTI women. We identified 22 differentially abundant taxa (FDR $<0.25)$ at lower taxonomic levels, 16 of which were depleted in rUTI women (Table S3; Figure 2b), including Faecalibacterium as previously reported ${ }^{9}$.

Several of the taxa reduced in the rUTI gut, including Faecalibacterium, Akkermansia, Blautia and Eubacterium hallii, are associated with short chain fatty acid (SCFA) production, including propionate and butyrate, which exert an anti-inflammatory effect in the gut through promotion of the intestinal barrier function and immunomodulation ${ }^{20,21}$. Blautia was additionally identified as the only taxon significantly depleted at the time of UTI relative to non-UTI samples (FDR=0.01). Cumulatively, SCFA producers, particularly butyrate producers, were significantly less abundant in rUTI women ( $p=0.001$ ) (Figure 2d; Figure S2). Four KEGG Orthogroups ${ }^{22}$ representing components of butyrate production pathways were significantly reduced across the rUTI cohort (Table S4). Functional analysis with HUMAnN2 ${ }^{23}$ additionally revealed distinctions between the cohorts. These included pathways associated with sugar degradation and biosynthesis of metabolite intermediates and amino acids, which were depleted in the rUTI cohort (Table S5), many of which were also found to be differentially abundant in a study of irritable bowel syndrome (IBS) patients with sugar malabsorption ${ }^{24}$.

The loss of gut microbial richness, diversity, and butyrate-producing bacteria that we observed is also a hallmark of exposure to broad spectrum antibiotics, including ciprofloxacin ${ }^{25-27}$, which was used to treat more than a third of UTIs in our study. Thus, we sought to determine whether antibiotic exposure similarly had an impact on the gut microbiome of women in this study, and whether antibiotic effects may contribute to the observed shifts in microbiome composition in rUTI women ('rUTI dysbiosis'). Though antibiotic exposure in the previous two weeks was 
associated with a significant reduction in microbial richness $(p=0.05)$, this antibiotic-mediated loss of richness was not sustained. Samples taken 2-6 weeks after antibiotic exposure were not significantly different from baseline levels $(p=0.2)$. Furthermore, we saw no association between the reported number of antibiotic courses and average richness (Figure 2c), and no differences in the overall gut microbiome stability between cohorts despite more frequent antibiotic treatment among UTI women (Figure S3). While low numbers of antibiotic exposures prevented a robust comparison of the effect by antibiotic class, we observed no differences in richness or in the abundance of butyrate producers between rUTI women with different antibiotic exposures (Figure S4). While we did not detect a lasting impact from individual antibiotic courses, it is still possible that repeated antibiotic use over multiple years may have contributed to the observed rUTI dysbiosis.

\section{rUTI gut dysbiosis shares broad similarities with inflammatory bowel disorders and may indicate low-level gut inflammation}

The depletion of butyrate-producing taxa and microbial richness, key characteristics of rUTI dysbiosis, are also observed in other gut inflammatory conditions including nosocomial diarrhea ${ }^{28}$, IBS ${ }^{29}$ and inflammatory bowel disease (IBD) ${ }^{20}$, particularly Crohn's disease (CD) ${ }^{30}$, and thus may be indicative of gut inflammation in rUTI women. While IBD is a multifactorial disorder for which the causative role of gut microbes is still incompletely understood ${ }^{31}$, mouse models have helped to demonstrate a causal relationship between gut dysbiosis and gut inflammation 32. To further explore the overlap between the shifts observed in IBD and rUTI guts compared to controls, we compared our data to longitudinal gut microbiome data from adults with and without IBD as part of the Human Microbiome Project 2 (HMP2) study ${ }^{33}$, which shared the same extraction and sequencing protocols as our study (Methods). Relative to each study's control group, we found that the ten most significantly depleted species in the rUTI gut, including butyrate producers $F$. prausnitzii and $E$. hallii, were also depleted in the IBD gut. We further observed a significant overall correlation in the estimated change of species-level abundances associated with rUTI and IBD (Figure S5), suggesting more general similarities.

There were also some notable differences. Bacteroides, significantly elevated in the rUTI group, did not differ between cohorts in the HMP2 study (Figure S5), and were also decreased among IBD patients in other studies ${ }^{34}$. E. coli was significantly elevated in IBD patients in the HMP2 study, but showed no difference in average relative abundance between our cohorts (Figure 2e). Indeed, diminished Bacteroides alongside elevated Enterobacteriaceae was also observed in patients with nosocomial diarrhea ${ }^{28}$. Diarrhea, also a symptom of IBD, is associated with reduced gut transit time, which has been shown to enrich for organisms common in the upper gastrointestinal tract, including Enterobacteriaceae ${ }^{35}$, at the expense of anaerobic organisms such as Bacteroides ${ }^{36}$. As such, rUTI women with low-level inflammation and no diarrhea may lack the depletion of Bacteroides and elevation of Enterobacteriaceae observed in other inflammatory, diarrhea-associated conditions. It is also possible that the considerable differences in treatment regimens for these conditions; i.e. antibiotics vs. anti-inflammatories, contribute to divergences of a common underlying inflammatory signal. 


\section{Differences in host immune response may dictate susceptibility to UTI}

Beyond inflammatory bowel disorders, rUTI dysbiosis also shares similarities with immunological syndromes affecting distal sites. For example, depletion of butyrate producers has been associated with rheumatoid arthritis, a systemic autoimmune disease which can be partially ameliorated in animal models with oral butyrate supplementation ${ }^{37,38}$. Patients with chronic kidney disease (CKD) also exhibit a similar dysbiosis to rUTI women, including reduced levels of Parasutterella and Akkermansia, the latter of which has been found to be inversely correlated with interleukin-10 levels, an anti-inflammatory cytokine ${ }^{39}$. We hypothesized that rUTI dysbiosis may also have an immunomodulatory role, potentially eliciting a differential immune response to bacterial invasion of the bladder. Thus, we broadly explored immunological biomarkers from blood samples collected at enrollment and at UTI, quantifying (i) a Luminex panel of human cytokines, chemokines, and growth factors involved in inflammation and T cell activation, and (ii) cell types and the transcriptional activity of peripheral blood mononuclear cells (PBMCs) (Methods).

Of the 39 analytes included in the Luminex panel, one chemokine, plasma eotaxin-1, was observed to be higher in rUTI women vs. control women at enrollment. Plasma eotaxin-1 is associated with intestinal inflammation ${ }^{40}$, and levels of eotaxin-1 are increased in colonic tissue of patients with active IBD versus healthy controls ${ }^{41}$. Subsequent human eotaxin-1 ELISAs validated these results, highlighting an additional link to dysbiosis-driven perturbation of the immune state, though, since this result did not hold after adjusting for race, we could not rule out potential demographic confounders. Eotaxin-1 was also higher in blood plasma of rUTI women at the time of UTI vs. enrollment ( $p=0.04$; Figure S6a).

Although our relatively small cohort size provided limited statistical power for such crosssectional comparisons, PBMC RNA-Seq analyses revealed two differentially expressed genes (FDR < 0.1), ZNF266 and the long non-coding RNA LINC00944, were upregulated in the PBMCs of the rUTI cohort compared to controls (Table S6). ZNF266 has been previously linked to urological health, identified as a potential PBMC biomarker for overactive bladder and incontinence in women ${ }^{42}$. LINC00944 has been associated with inflammatory and immunerelated signaling pathways as well as tumor invading $T$ lymphocytes in breast cancer, and markers for programmed cell-death ${ }^{43}$. A CIBERSORT analysis of cell types based on transcriptional profiles revealed that resting NK cells were significantly reduced at the time of UTI relative to baseline levels ( $p=0.02$; Figure $S 6 b$ ). NK cells help suppress bladder infection by UPEC in mice ${ }^{44}$, so the loss of NK cells in the periphery may suggest a migration to the bladder at time of rUTI.

\section{rUTI and control cohorts showed similar E. coli dynamics in both the gut and the bladder}

Previous work has implicated gut dysbiosis and a depletion of butyrate-producing bacteria in enhanced susceptibility to gut colonization by pathogens, including Salmonella ${ }^{45}$ and C. difficile ${ }^{46}$. While we could not quantify absolute species abundances, we observed no significant 
difference in the average relative abundance of $E$. coli between cohorts in this study (Figure 2e) suggesting that the rUTI dysbiotic gut is no more hospitable to $E$. coli colonization than controls. We considered the possibility that a temporal increase, or bloom, in $E$. coli relative abundance is a rUTI risk factor. Of the samples collected in the 14 days preceding an E. coli UTI, 75\% exhibited E. coli relative abundance at or above average levels in the gut (Figure S7). However, elevated E. coli levels were not predictive of UTIs; none of the 22 E. coli blooms (here, defined as $E$. coli relative abundance more than 10 -fold higher than the intra-host mean) occurred in the two weeks prior to UTI diagnosis. Thänert et al. identified intestinal blooms of uropathogens preceding some UTIs, but similarly noted that blooms often occurred in the absence of infection 7 , leading us to conclude that elevated levels of $E$. coli may facilitate transfer to the bladder, but such gut blooms rarely manifest in infection. However, without frequent urine collection, we cannot rule out asymptomatic bladder colonization on such occasions.

Though we did not detect differences in E. coli species dynamics, the E. coli species encompasses considerable phenotypic and phylogenetic diversity. E. coli strains are broadly delineated into evolutionarily divergent phylogroups ${ }^{47}$ with UPEC strains most commonly arising from phylogroups $B 2$ and $D^{4,48}$. We hypothesized that rUTI dysbiosis may manifest in a qualitatively different $E$. coli population in the gut, contributing to increased susceptibility to rUTI. In order to explore E. coli strain-level diversity within stool metagenomes, we applied StrainGE, a tool suite to characterize the strain(s) of a given, low-abundance species within complex microbial communities ${ }^{49}$ (Methods). Patterns of strain carriage were broadly similar in the rUTI (Figure 3) and the control (Figure S8) cohorts. Both the number of strains per sample and the phylogroup distribution were comparable between cohorts (Figure 4, Figure S9). While the majority of identified E. coli strains (62\%) were transient, observed in one sample only, $22 \%$ were 'persistent', observed in at least one quarter of their carrier's samples. Persistent strains were more likely to originate from phylogroups $B 2$ and $D$ than others $(p=0.01)$, regardless of cohort, and were even slightly more common in control women $(O R=2.1(0.9,5.2), p=0.1)$, at odds with the hypothesis of differential colonization resistance to phylogroups associated with UPEC between cohorts.

Given the similar E. coli strain dynamics observed in the gut between cohorts, we applied StrainGE to all urine samples, seeking to elucidate differences in strain dynamics in the bladder. We found that $79 \%(11 / 14)$ of $E$. coli UTIs were caused by phylogroup B2 $(n=7)$ or $D(n=4)$ strains (Table S2), approximately in line with previous studies ${ }^{4}$. Of the 24 healthy enrollment urine samples yielding sufficient bacterial DNA to be sequenced and profiled (Table S7), we detected $E$. coli strains in 54\% (13/24), including over half of samples (7/13) from control participants, despite the absence of symptoms. All but one of these strains also belonged to phylogroups B2 and D. Control urines carried E. coli strains that were phylogenetically similar to UTI-causing strains based on StrainGE predictions (Figure 4; Methods), despite divergent clinical outcomes.

Mapping urine metagenome assemblies to a curated virulence factor database showed that UTI-causing strains were enriched in virulence factors (including iron uptake systems (sit, chu, iro, ybt operons), colibactin (clb), and type 6 secretion systems) relative to an $E$. coli species- 
wide database, though many of these were also present in the one urine sample from a control participant for which we had sufficient coverage to assess gene content (Methods, Table S8). This apparent transition of a likely urovirulent strain to the bladder of healthy women without eliciting UTI symptoms is consistent with previous studies which have been unable to identify genetic markers of urovirulence in mice ${ }^{48}$, or consistently discriminate between UTI and asymptomatic bacteriuria strains in women ${ }^{50}$. Nevertheless, the divergence in clinical outcomes after bacterial invasion of the bladder may still arise due to phenotypic differences in $E$. coli strains reaching the bladder that are not readily apparent in genome comparisons. It is possible that rUTI dysbiosis could have an impact on UPEC gene expression; it has been shown that higher SCFA levels are associated with down-regulation of $E$. coli virulence factors including fimbrial and flagellar genes ${ }^{51}$, however, such transcriptional analyses fall outside the scope of this study.

\section{rUTI strains colonize the gut and long-term clearance was not achieved by antibiotic treatment}

While it is well known that UTIs are most commonly caused by UPEC resident in the gut, longitudinal dynamics of these strains within the gut are less well understood, despite the importance of such insights into developing rUTI prophylaxis. As such, we applied StrainGE to all urine samples to identify UTI-causing strains and their corresponding gut dynamics, in particular at the time of UTI, as well as after antibiotic exposure.

Four rUTI women suffered multiple confirmed E. coli UTIs, though only one was a same strain recurrence (individual 8) (Figure 3), suggesting that rUTIs in this study population were not frequently seeded by a monoclonal UPEC reservoir. Comparisons of sequence data from urine samples and cultured rectal swabs collected during UTI clinic visits revealed that nearly all $(11 / 12)$ E. coli UTIs, for which we had corresponding same-day rectal swabs, contained the same UTI strain, underscoring frequent transmission between the gut and bladder. Though not a perfect proxy for native gut relative abundances, the dominant $E$. coli strain in 4 of the rectal swab outgrowths was not the UTI-causing strain, suggesting that some UTIs may be caused by minority strains. Only one UTI (individual 5, Figure 3) was caused by an E. coli strain never observed in any other sample from that individual. This phylogroup B1 UTI-causing strain likely arose from a source other than the gut, such as the urinary tract or the vagina, which have been implicated as UPEC reservoirs ${ }^{7,52}$.

We anticipated that antibiotic exposure - particularly ciprofloxacin - would impact gut carriage of E. coli strains, and may explain the lower frequency of persistent colonizers in the rUTI group. Indeed, E. coli strains were detected by StrainGE significantly less frequently in stool samples provided in the two weeks following antibiotic use $(\mathrm{OR}=0.3(0.13,0.68) ; p=0.004)$. However, many strains apparently cleared by antibiotics were observed again at later time points; in fact, none of the UTI-causing strains observed in the gut was permanently cleared following antibiotic exposure. While low-level persistence that is undetectable based on the sequencing data is a possibility, we plated a subset of post-treatment stool samples onto MacConkey agar to culture E. coli. In many cases, we observed no growth, further suggesting the absence of these strains 
for a period of time post-treatment (Table S9). Furthermore, antibiotic susceptibility profiling of 12 UTI-causing strains isolated from proximate stool samples demonstrated that the majority were susceptible to the antibiotics to which they were exposed (Table S10). While a single stool sample is not completely representative of the gut microbiota, our evidence suggests that UTIcausing strains may be frequently reintroduced to the gut from alternative sources following antibiotic clearance of the bladder and gut.

\section{rUTI is linked to perturbations of the gut-bladder axis, highlighting potential for novel therapeutics}

Our study design, data collection and culture-independent metagenomic sequencing approach allowed us to characterize dynamics of the gut-bladder axis in healthy and rUTI women. We propose that rUTI susceptibility is dependent, in part, on perturbation of the gut-bladder axis, which represents a previously undescribed syndrome, comprising gut dysbiosis and differential host immunology. We show that, compared to healthy controls, women suffering rUTI exhibited gut dysbiosis characterized by depleted levels of butyrate-producing bacteria and diminished microbial richness. This dysbiosis did not appear to impact $E$. coli dynamics within the gut; relative abundances and strain types were similar between cohorts, suggesting that gut carriage of urovirulent bacteria in itself is not a risk factor for rUTIs. Notably, E. coli was commonly identified in the urine of healthy women, including strains arising from UPEC-associated clades and harboring similar virulence factors. Based on our observations, rUTI gut dysbiosis is consistent with low-level gut inflammation, and is reminiscent of other disorders in which microbiome-mediated immunomodulation may play a role in disease severity. We identified several immunological differences despite the limited size of our cohorts, supporting the hypothesis of a differential host immunology. Our findings warrant further investigations to explore microbiome-host mucosal immune interactions in the context of rUTI susceptibility.

While identifying the origins of rUTI dysbiosis is outside the scope of this study, repeated antibiotic exposure is a highly plausible mechanism through which this dysbiosis is maintained. Whether the observed dysbiosis is the direct result of long-term antibiotic perturbation is unclear, and the relatively short study period precluded us from establishing such causal relationships here. In addition to the potentially detrimental impact of antibiotic use on the gut microbiota, we found that treatment also failed to clear UTI-causing strains from the gut in the long term. As such, mitigation of rUTI risk via manipulation of the E. coli population appears likely to require long-term therapy to overcome repeated introductions from non-gut reservoirs. rUTI treatment protocols targeting UPEC strains in the gut while resulting in minimal disruption to the remaining gut microbiota, such as small molecule therapeutics ${ }^{53}$, may offer improved long-term prospects. While more evidence is required to fully characterize the causal mechanisms between dysbiosis and infection, our work highlights the ineffectiveness and potential detrimental impact of current antibiotic therapies, as well as the potential for microbiome therapeutics (e.g. fecal microbiota transplants ${ }^{10}$ ) to limit infections via restoration of a healthy bacterial community in the gut. 


\section{Methods}

\section{Study design \& sample collection}

\section{Enrollment}

This study was conducted with the approval and under the supervision of the Institutional Review Board of Washington University School of Medicine in St. Louis, MO. Women from the St. Louis, MO area reporting three or more UTIs in the past 12 months were recruited into the rUTI study arm, while women with no history of UTI (at most one UTI ever) were recruited into the control arm via the Department of Urological Surgery at Barnes-Jewish Hospital in St. Louis, MO. We excluded women who: i) had inflammatory bowel disease (IBD) or urological developmental defects (e.g., ureteral reflux, kidney agenesis, etc.), ii) were pregnant, iii) take antibiotics as prophylaxis for rUTI, and iv) were younger than 18 years or older than 45 at the time of enrollment. All participants provided informed consent. A total of 16 control and $15 \mathrm{rUTI}$ women were recruited to the study; 14 women in each cohort completed the entire study collection protocol. Participants who did not complete the study were included in cohort-level comparisons, but excluded from longitudinal analyses.

\section{Sample collection \& storage}

Participants provided blood and urine samples, as well as rectal swabs, at the initial clinic visit. UTIs were diagnosed during clinic visits; additional UTIs (not presenting at the study clinic) were inferred based on symptoms and antibiotic consumption reported in the monthly questionnaire. Women visiting the clinic during the study with UTI symptoms provided rectal swabs, blood and urine samples, and were requested to submit stool samples as soon as possible (within 24 hours) after the clinic visit, as well as at a two week follow-up time point.

All participants provided monthly stool samples for 12 months. Samples were collected at home, and submitted via mail. Questionnaires were completed with all monthly and clinical sample collections; these captured self-reported antibiotic and drug use, dietary intake, sexual intercourse and UTI symptoms.

\section{Sample processing}

\section{Blood sample preparation}

A total of $15 \mathrm{~mL}$ of blood was collected from each patient during initial enrollment and UTI visits. The blood was stored on ice for less than 30 minutes and then mixed with an equal amount PBS with $2 \%$ fetal bovine serum (FBS). Peripheral blood mononuclear cell (PBMCs) were then isolated using SepMate PBMC isolation tubes (Stemcell Technologies) with Ficoll-Paque PLUS density gradient medium (Cytiva). Serum was collected during the PBMC isolation process and stored at $-80 \mathrm{C}$ until use. PBMCs were washed with PBS plus $2 \% \mathrm{FBS}$ and pelleted via centrifugation at $10,000 \times \mathrm{g}$ at room temperature for 5 minutes. PBMC cell pellets were then flash frozen and stored at $-80 \mathrm{C}$ until RNA extraction. 


\section{Rectal swab and urine preparation}

Rectal swabs were collected in the clinic and stored on ice for less than 30 minutes. Rectal swabs were washed in $2 \mathrm{~mL}$ of PBS. $1 \mathrm{~mL}$ of PBS was centrifuged at $10,000 \mathrm{xg}$ at room temperature for 2 minutes and the PBS supernatant was removed. The bacterial/fecal pellet was then flash frozen and stored at $-80 \mathrm{C}$ until DNA extraction. The remaining $1 \mathrm{~mL}$ was then used to make serial dilutions and then plated on both Luria Broth (LB) and MacConkey agar and incubated overnight at $37 \mathrm{C}$ to quantify colony forming units (CFUs). After bacterial enumeration, bacteria from MacConkey and LB plates were scraped to collect bacterial outgrowths. Bacterial cells were washed with PBS, pelleted at $10,000 \times \mathrm{g}$ at room temperature for 2 minutes, flash frozen and then stored at $-80 \mathrm{C}$ until DNA extraction.

Mid-stream urine samples were collected in sterile containers and stored on ice for less than 30 minutes. $10 \mathrm{~mL}$ of urine was centrifuged at $10,000 \mathrm{xg}$ at room temperature for 5 minutes. The resulting pellet was washed in PBS, pelleted again, and then flash frozen and stored at -80C until DNA extraction. $1 \mathrm{~mL}$ of urine was used to make serial dilutions and then plated onto both LB and MacConkey and incubated overnight at 37C to enumerate CFUs. After outgrowth, the plates were scraped to collect bacterial colonies, which were then washed with PBS, pelleted at $10,000 \times \mathrm{g}$ at room temperature for 2 minutes, flash frozen and then stored at -80C until DNA extraction.

\section{RNA Extraction - PBMCs}

RNA was extracted from stored PBMCs using TRIzol Reagent (cat. no. 15596-026 and 15596018; Life Technologies), according to the manufacturer's protocol. Briefly, $0.75 \mathrm{~mL}$ of TRIzol was added per $0.25 \mathrm{~mL}$ of sample and cells were lysed by several rounds of pipetting. Samples were incubated for five minutes at room temperature. Chloroform was added to the samples at the recommended concentration and samples were incubated shaking for 15 seconds and set to rest for 2-3 minutes at room temperature. After incubation, samples were centrifuged at $12,000 \times g$ for 15 minutes at $4 \mathrm{C}$. The aqueous phase was collected for RNA isolation. RNA was precipitated using $100 \%$ isopropanol and incubated at room temperature for 10 minutes, followed by centrifugation at $12,000 \times g$ for 10 minutes at $4 \mathrm{C}$. The precipitated RNA was washed according to the protocol using $75 \%$ ethanol and resuspended in RNase-free water. Extracted RNA was stored at $-80 \mathrm{C}$ until further use.

\section{DNA Extraction - Rectal Swabs \& Urine}

DNA was extracted from rectal swabs and urine samples plated on MacConkey agar using the Wizard Genomic DNA Purification Kit (Promega), according to the manufacturer's protocol. Briefly, samples were resuspended in $600 \mathrm{uL}$ of Nuclei Lysis solution and incubated at $80 \mathrm{C}$ for five minutes, then cooled to room temperature. RNase solution was added to samples and incubated for 15 minutes at 37C, then cooled to room temperature. $200 \mathrm{uL}$ of Protein Precipitation solution was added to the RNase-treated sample, vortexed for 20 seconds, and incubated on ice for 5 minutes. After incubation, samples were centrifuged for 3 minutes at $16,000 \times g$ and the supernatant was transferred to a $1.5 \mathrm{~mL}$ microcentrifuge tube containing 600 
uL of isopropanol. Samples were gently mixed and centrifuged for 2 minutes at $16,000 \times g$. The supernatant was removed and the DNA pellet was washed with $70 \%$ ethanol. Samples were centrifuged for 2 minutes at 16,000 $\mathrm{g}$, ethanol was aspirated and DNA pellet was air-dried for 15 minutes. The DNA pellet was rehydrated with DNA Rehydration solution and incubated at $65 \mathrm{C}$ for 1 hour. Extracted DNA was stored at $4 \mathrm{C}$ for short-term storage and at $80 \mathrm{C}$ for long-term storage until further use.

\section{WMS sequencing \& sequence data processing}

Libraries were constructed from extracted DNA from stool, urine, rectal swabs, and plate scrapes using the NexteraXT kit (Illumina). Then, libraries were sequenced on a HiSeq 2500 (Illumina) in 101 bp paired-end read mode and/or a HiSeq X10 (Illumina) in 151 bp paired-end read mode. Sequence data was then demultiplexed. Samples that were sequenced multiple times on different runs were pooled together. Reads were processed with KneadData (v0.7.2, https://huttenhower.sph.harvard.edu/kneaddata/) to remove adapter sequence and trim low base qualities (with Trimmomatic), as well as to remove human-derived sequences (by aligning to human genome with bowtie2).

\section{Luminex assays}

Custom Luminex magnetic bead assay kit was obtained from R\&D systems (product LXSAHM). Analytes from Human Inflammation and Human T Cell Response panels were chosen for the custom kit of 39 analytes: CXCL1/GROalpha, IL-1alpha, M-CSF/CSF1, LIF, LTalpha/TNF-b, MIF, APRIL, CCL11/Eotaxin, CCL4/MIP-1b, CXCL8/IL-8, IFN-g, IL-1b, IL-11, IL-13, IL-17A, IL18, IL-21, IL-27, IL-31, IL-4, IL-6, MMP-1, TNF-a, BAFF/BLyS, CCL2/MCP-1, CX3CL1/Fractalkine, CXCL5/ENA-78, GM-CSF, IL-10, IL-12p70, IL-15, IL-17E/IL-25, IL-2, IL22, IL-28A/INF-12, IL-33, IL-5, IL-7, MMP-3. Detection of the analytes in human plasma samples was performed using the Curiox DropArray system for miniaturization of magnetic bead multiplex kits. Plasma samples were diluted 2-fold for the assay. Results were read and quantified using a BioPlex multiplex plate reader and Microplate Manager software.

\section{Eotaxin ELISA}

Plasma eotaxin (CCL11) levels from rUTI and control patients were measured using the Eotaxin (CCL11) Human Simple Step ELISA kit (cat. no. ab185985; Abcam), according to the manufacturer's protocol. Briefly, plasma samples were diluted into sample diluent and $50 \mathrm{uL}$ of sample and $50 \mathrm{uL}$ of antibody cocktail were added to 96 well plate strips. Plates were sealed and incubated shaking for one hour at room temperature. Wells were washed three times with $1 \mathrm{x}$ wash buffer and inverted to remove excess liquid. $100 \mathrm{uL}$ of TMB substrate was added to each well; plates were covered to protect from light and incubated shaking for 10 minutes. Stop solution $(100 \mathrm{uL})$ was added to each well and plates were incubated shaking for one minute. The $\mathrm{OD}_{450}$ was measured and recorded to determine the concentration of Eotaxin in $\mathrm{pg} / \mathrm{mL}$. 


\section{Sequence data analysis}

\section{Community profiling and metrics}

Bacterial community composition was determined using MetaPhIAn2 (v2.7.0 with db v20) ${ }^{54}$ on KneadData-processed sequences. Functional profiling was performed by HUMAnN2 (v2.8.1, database downloaded in October 2016) ${ }^{23}$ on KneadData-processed sequences. Diversity metrics and Bray-Curtis distances were derived from the MetaPhIAn2 relative abundance output using the vegan package in $\mathrm{R}$ [https://cran.r-project.org/web/packages/vegan/].

\section{PBMC RNASeq analysis}

Sequences from PBMC extracted mRNA were aligned to the human reference genome (hg19) using the STAR aligner ${ }^{55}$. Picard-Tools (https://broadinstitute.github.io/picard/) was used to mark duplicate reads. Read counts per gene were generated with subread featureCounts ${ }^{56}$. Read counts were normalized into Counts Per Million (CPM) using edgeR ${ }^{57}$. This normalized read count matrix was then used as input for CIBERSORT using the LM22 signature gene set 58. Results from CIBERSORT reported the relative abundance of 22 different immune cell types, including both PBMC and non-PBMC cell types, and it was used to remove three samples that were contaminated with $5 \%$ or greater of non-PBMC cell types. The CIBERSORT filtered set of samples was used to perform differential gene expression analysis using DESeq2 ${ }^{59}$. Baseline healthy control samples were compared to baseline rUTI samples. Due to limited sample numbers and potential confounding, we included only samples collected from caucasian women in this analysis. Results driven by single outlying data points were not considered.

\section{E. coli strain profiling}

In order to track E. coli strain dynamics we used Strain Genome Explorer (StrainGE) ${ }^{49}$. We applied the StrainGST module of StrainGE to identify representative E. coli strains in all stool, urine and rectal swabs, using an $E$. coli reference database generated from RefSeq complete genomes, as detailed in that paper. Strains mapping to the same representative reference genome in this database typically have an ANI of at least $99.9 \%$. To provide further evidence that same-strain calls from sample pairs from the same host were indeed matches, we ran the StrainGR module of StrainGE, which calculates alignment-based similarity metrics. We used benchmarked thresholds to determine strain matches; strain pairs with a common callable genome $>0.5 \%$, Jaccard gap similarity $>0.95$ and average callable nucleotide identity $>99.95 \%$ were deemed matches.

\section{Determination of UTI-causing strains}

Urine samples provided at the time of UTI diagnosis were plated on MacConkey agar. Sequence data was generated from DNA extracted from uncultured urine, and/or outgrowth on selective media. The cause of UTI was deemed to be the most abundant uropathogen, using outgrowth data where available, uncultured urine otherwise. Species were determined to be uropathogens based on UTI prevalence studies, e.g. '. 
medRxiv preprint doi: https://doi.org/10.1101/2021.11.15.21266268; this version posted November 16, 2021. The copyright holder for this preprint (which was not certified by peer review) is the author/funder, who has granted medRxiv a license to display the preprint in perpetuity.

It is made available under a CC-BY-NC 4.0 International license .

\section{Determination of virulence factors}

Urine metagenomes for which $E$. coli represented the dominant species were assembled using SPAdes ${ }^{60}$. To detect virulence factors in E. coli references (see StrainGST section above) and assembled genomes from study samples, we used the Virulence Factor Database (VFDB) for $E$. coli and the type 6 secretion system (T6SS) database (SecReT6) in genome-wide BLAST+ searches. Though VFDB contains T6SS genes, we removed them in favor of the T6SS-specific database for a T6SS-specific analytical pipeline. Other VFDB hits from blastn were filtered for $\geq 90 \%$ identity and $\geq 90 \%$ coverage. All $E$. coli genomes were separated by phylogroup for enrichment analysis, where Fisher's Exact test was used to determine the significance of virulence factor enrichment in a certain phylogroup. T6SS hits were filtered for $\geq 90 \%$ identity and $\geq 90 \%$ coverage and the system was considered present where at least 12 different adjacent T6SS genes were present. Again, an enrichment analysis was performed using Fisher's Exact test to determine the significance of T6SS presence in certain phylogroups.

\section{Statistical testing \& models}

\section{rUTI risk factors}

We used questionnaire responses to determine if any dietary or behavioral factors were associated with rUTI. We first compared the proportion of participants in each cohort who responded positively to binary variables (e.g. dairy consumption, alcohol etc. in the previous two weeks) in more than $50 \%$ or responses, and used a Fisher's Exact test to determine significance. We next fit mixed effects logistic regression models to determine temporal risk factors for UTIs. Samples collected within 3 days of UTI diagnosis were classified as 'time of UTI'; this binary variable was fit as a function of host (random effects term) and each dietary or behavioral response variable collected in the questionnaire. Variables with limited or no variance were excluded.

\section{Identifying differences at the cohort level and time of UTI}

We fit mixed effects linear regression models to compare the structure, diversity and function of the gut microbiome between cohorts. An arcsine square root transformation was applied to relative abundance values. Features (transformed relative abundances, diversity, microbial richness) were fit as a function of host (random effects term), cohort (categorical variable), and terms for antibiotic use and race (categorical variable) to adjust for potential confounding effects. To assess change in relative abundances at relevant timepoints, we also fit models including covariates for 'pre-UTI' (14 days preceding UTI diagnosis), 'time of UTI' (three days either side of UTI diagnosis), or post antibiotics ( $<14$ days post antibiotic exposure) as binary variables. All taxa with more than $10 \%$ non-zero values were fitted using the Ime4 function in R. Wald's test was used to derive raw $p$-values, which were adjusted for multiple hypothesis testing using Bonferroni-Hochberg correction at each taxonomic level.

The relative abundance of SCFA producers was additionally compared between cohorts; butyrate- and propionate-producing species were determined based on functional capacity to 
produce butyrate and propionate ${ }^{61}$. These species' relative abundances were then aggregated and compared as above.

We compared the stability of the microbiome between cohorts by assessing the distributions of within-host pairwise Bray-Curtis (BC) dissimilarities between individuals. Since rUTI women had, on average, slightly more frequent sampling than control women, due to the additional follow-up samples after UTI diagnoses, this metric may be biased towards smaller values in this cohort. However, we observed no significant trend between BC dissimilarity and time between samples, suggesting no detectable long-term trends. Furthermore, we detected no difference in the distribution of time-adjusted BC distances (BC divided by number of days between samples) between cohorts.

\section{IBD comparisons}

To compare rUTI dysbiosis to an IBD gut state, we downloaded MetaPhIAn2 output from the HMP2 study ${ }^{33}$, (ibdmdb.org). We extracted longitudinal samples from adults with IBD (diagnosis='UC' or 'CD') and non-IBD controls (diagnosis='nonIBD'). We fit linear mixed effects models with standardized relative abundances as a function of host (random effects term), race (race='white'; binary term) and recent antibiotic use. Fitted coefficients for the IBD and rUTI cohorts are then plotted in Figure S5.

\section{Acknowledgements}

This project has been funded in part with Federal funds from the National Institute of Allergy and Infectious Diseases, National Institutes of Health, Department of Health and Human Services, under Grant Number U19Al110818 to the Broad Institute.

We would like to acknowledge members of the Broad's Bacterial Genomics group and Hera Vlamakis for helpful conversations. We thank Brian Haas for assistance with PBMC RNA-Seq analysis as well as the Multi-Omics Core and Genomics Platform at the Broad Institute for sample processing and data generation.

\section{Author contributions}

Study design HLS, KWD, SJH, AME

Study coordination HLS, KB, SBC, AK

Experiments performed HLS, JSP, CLPO, VLM, AEP

Data analysis CJW, HLS, TJS, LRvD, RAB, BO, BJH, CAD, WCC

Consultation and supervision of analyses BJW, ALM, TJH, TMH, ALK, HHL, KWD, SJH, AME

Prepared the original draft CJW, ALM, KWD, SJH, AME

Review and approval of the final manuscript was provided by all authors. 


\section{Competing interests}

The authors declare no competing interests.

\section{Data availability}

Sequence data are available from the Sequence Read Archive under Bioproject PRJNA400628.

\section{Figures}

A

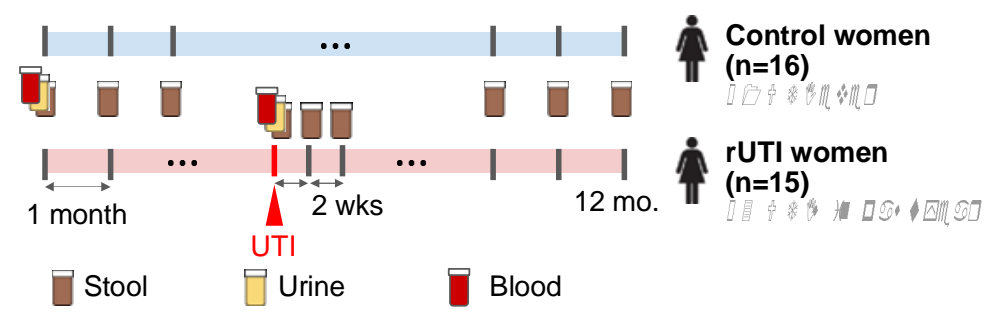

B

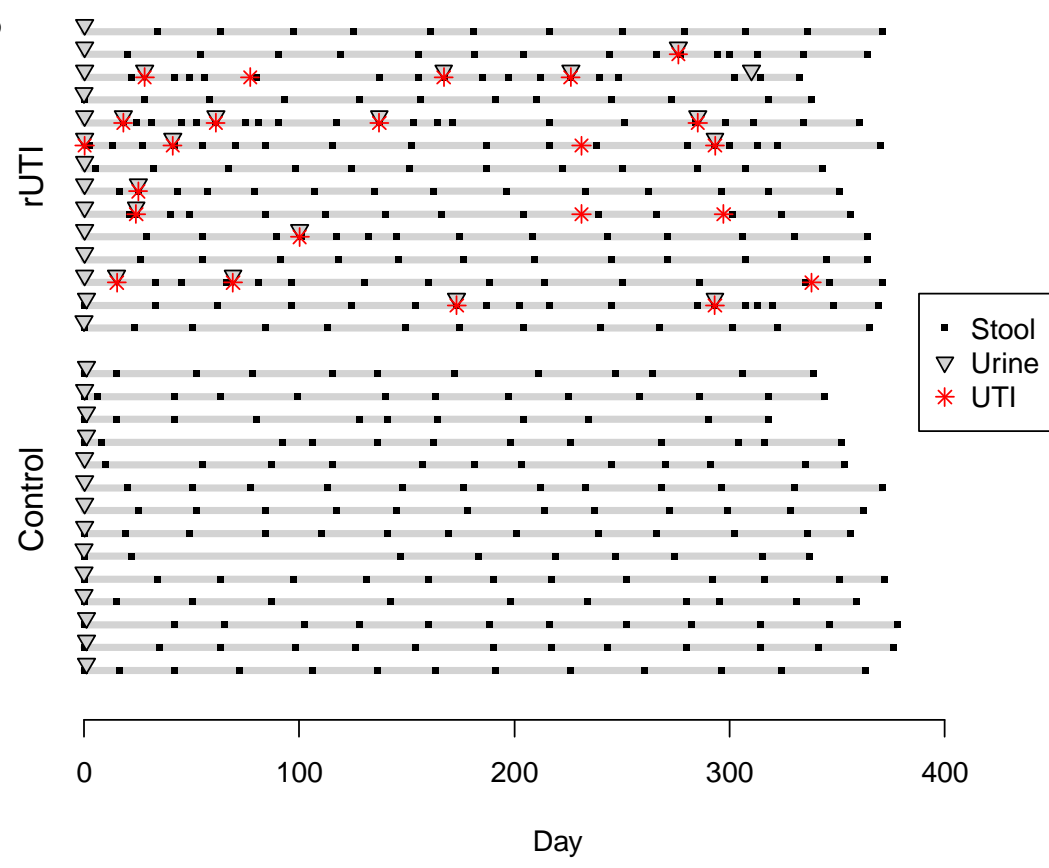

Figure 1. Study design and sample collection for the UMB study. (a) Stool samples were collected monthly from rUTI and control patients. Stool, urine and blood plasma samples were collected upon enrollment and subsequent UTI clinic visits. Biweekly stool samples were requested following UTI diagnoses. (b) Stool and urine samples collected from all rUTI and control participants who completed the study protocol. Red symbols denote diagnosed and inferred UTI events. 

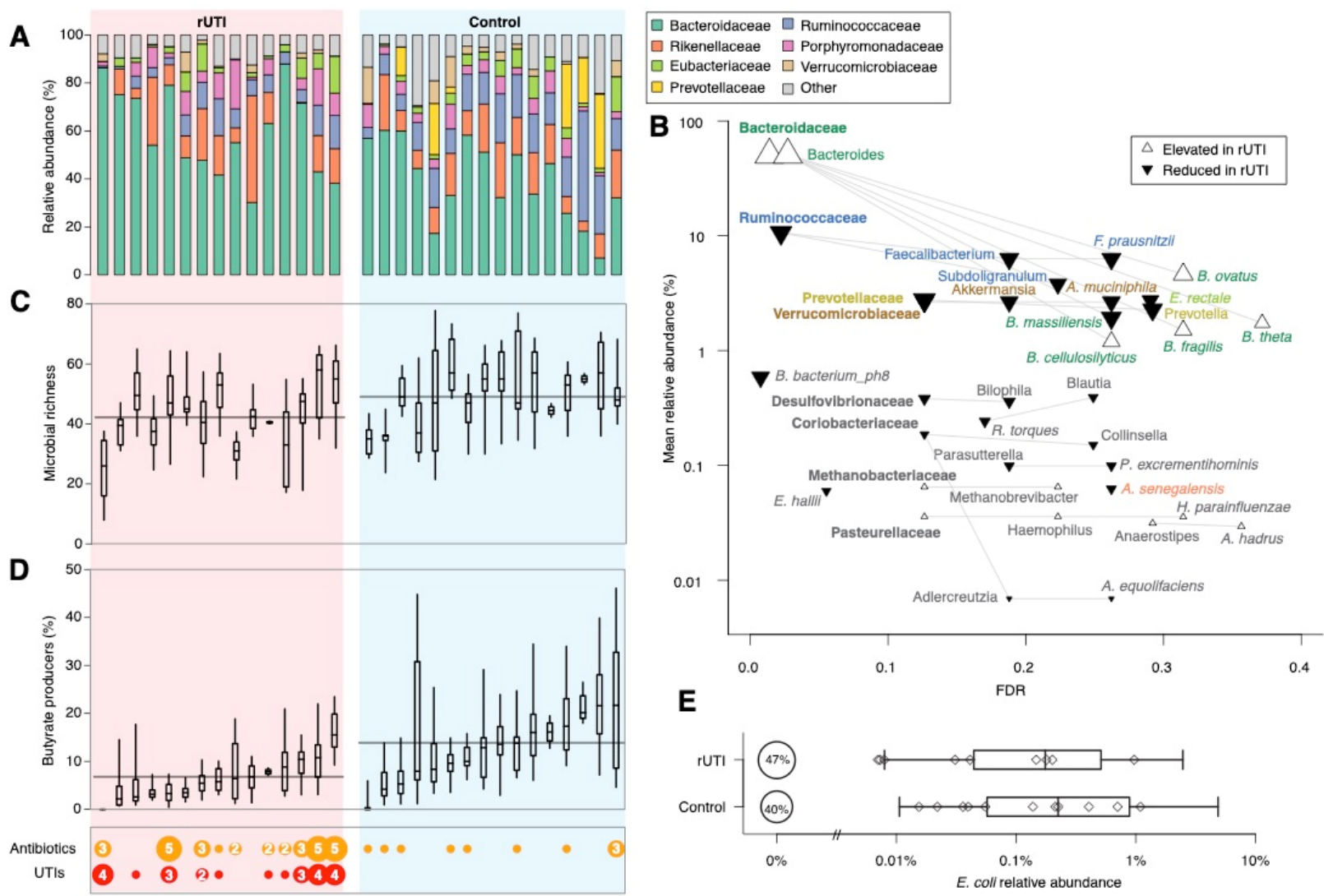

Figure 2. rUTI women have a distinct gut microbiome. (a) Average relative abundances of bacterial families for each patient in the rUTI (left) and the control (right) cohorts. (b) Significance and rUTI group effect size for selected taxa. Each point represents one taxon; its effect size and direction (symbol) for rUTI vs. control, false discovery rate (FDR) and mean relative abundance across all samples. Taxonomic relationships are represented by lines. FDR values calculated independently at each taxonomic level. Bold, regular and italic text denote family, genus and species levels (c) Microbial richness distributions and (d) cumulative relative abundance of butyrate-producing species for each study participant. Box plots depict IQR and central $95 \%$ quantile for each individual. Horizontal lines represent group-level mean of individual means. Antibiotic use and UTI occurrence for each study participant is shown at the bottom left; symbol size and numerals denote the number of UTIs/reported antibiotic courses. (e) Relative abundance of $E$. coli in each cohort. Symbols denote median relative abundances of individual patients; box plots denote the interquartile range and $95 \%$ central quantile of nonzero values. 
medRxiv preprint doi: https://doi.org/10.1101/2021.11.15.21266268; this version posted November 16, 2021. The copyright holder for this preprint (which was not certified by peer review) is the author/funder, who has granted medRxiv a license to display the preprint in perpetuity.

It is made available under a CC-BY-NC 4.0 International license .

A

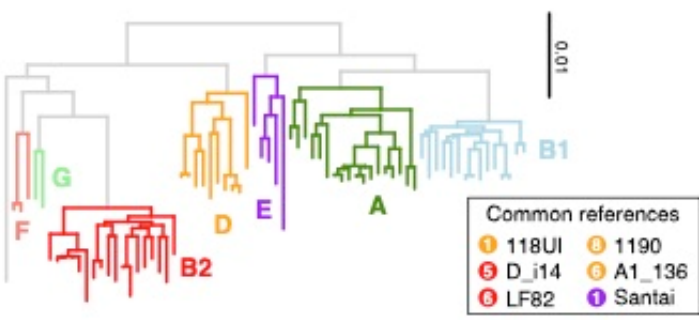

B

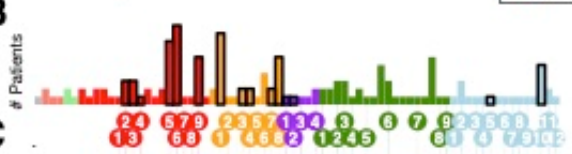

D

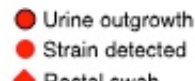

Strain detected

Rectal swab

No strains presen

| UTI diagnosis
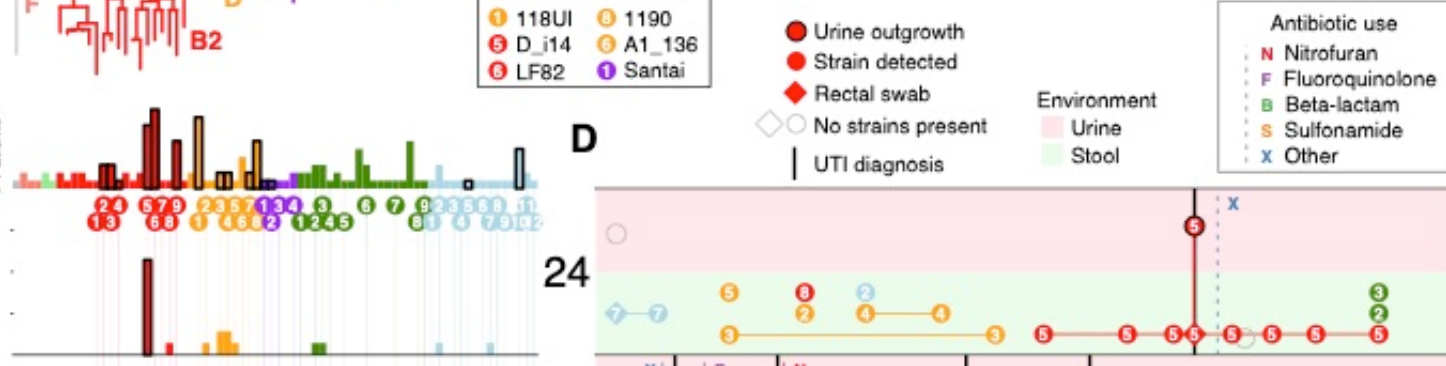

24

o
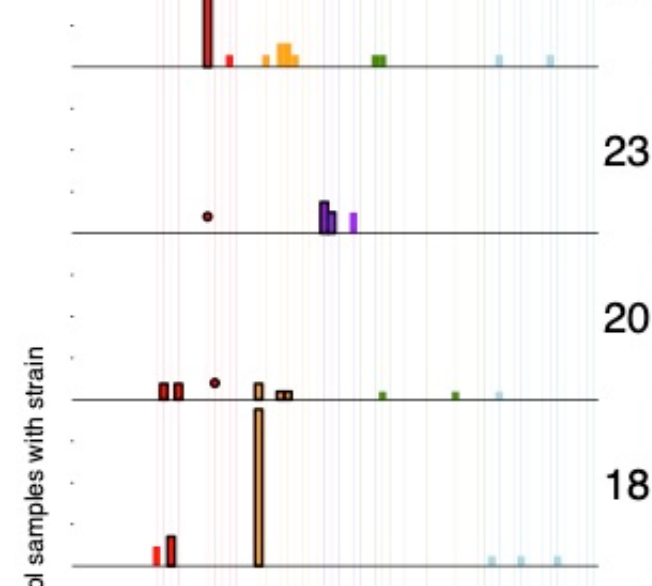

20

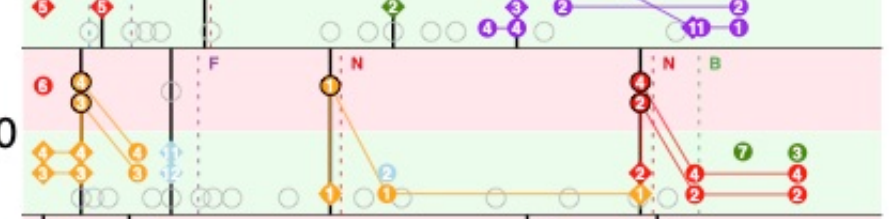

18

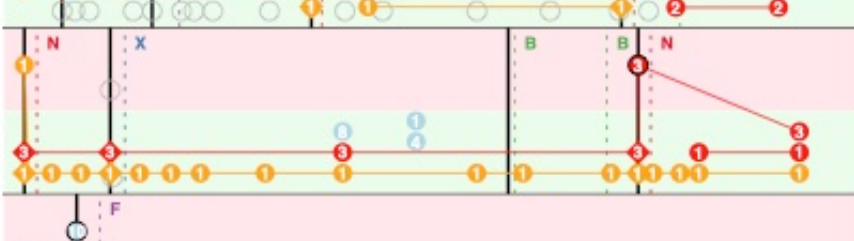

15

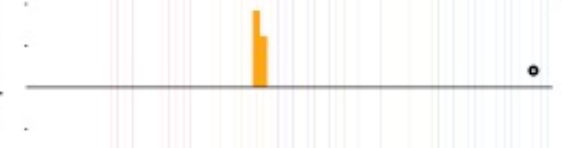

12
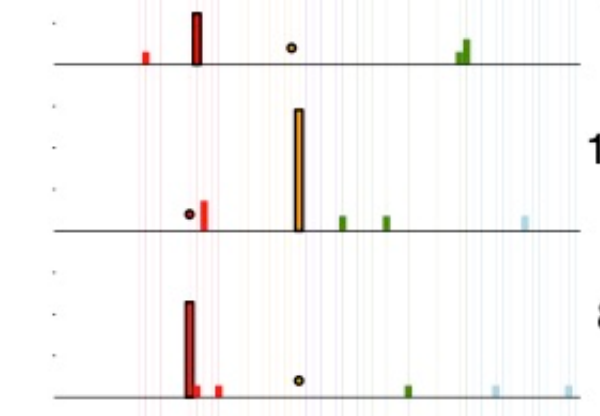

8

11
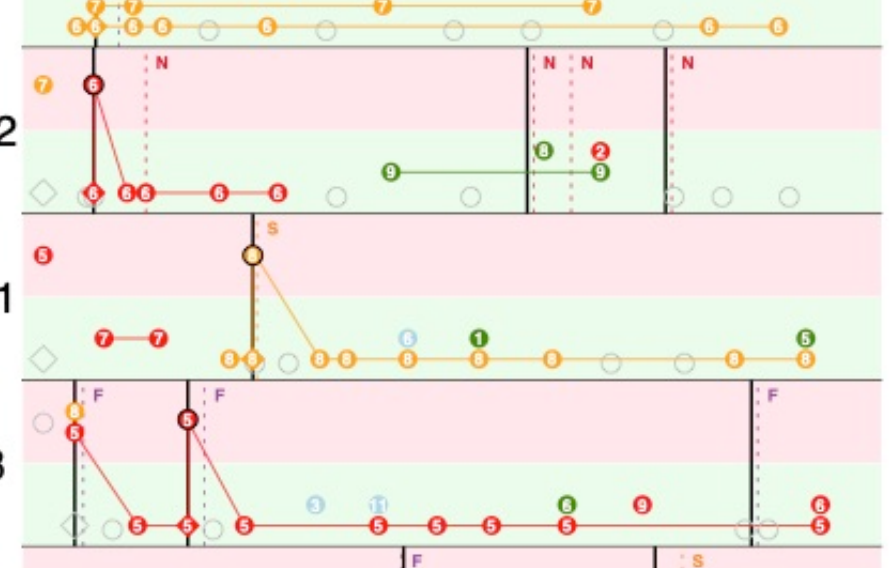

5

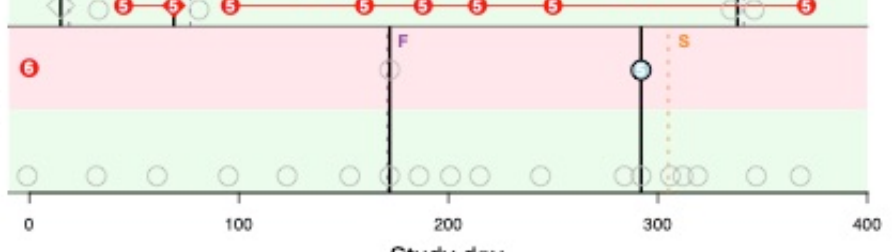

Figure 3. Frequent gut-bladder transmission and strain persistence in rUTI patients.

Strain dynamics within all participants with E. coli UTIs. (a) Phylogenetic tree comprising strains called by StrainGE across all stool and urine samples, colored by phylogroup. (b) Number of unique patients with at least one strain observation, bolded if observed in any urine sample. Each strain identified in rUTI patients is uniquely identifiable by the phylogroup (color) and ID 
medRxiv preprint doi: https://doi.org/10.1101/2021.11.15.21266268; this version posted November 16, 2021. The copyright holder for this preprint (which was not certified by peer review) is the author/funder, who has granted medRxiv a license to display the preprint in perpetuity.

It is made available under a CC-BY-NC 4.0 International license .

(numeral) indicated below. (c) Each panel represents one patient and the proportion of their stool samples in which each strain was identified; bold outlines if also observed in that patient's urine. Dots represent observation in urine samples only. (d) Each panel represents longitudinal strain dynamics within one patient. Numerals refer to strain identifiers in (b). All strains are connected to their most recent previous observation in either fecal samples (green background), urine samples (pink). Diamonds denote clinical rectal swabs. Strains identified in urine outgrowth depicted if available (bold); otherwise raw urine strains are shown. Stool, urine or samples with no detected E. coli strains represented by open grey symbols. Vertical dashed lines represent self-reported antibiotic use, solid black lines denote UTI events. 
medRxiv preprint doi: https://doi.org/10.1101/2021.11.15.21266268; this version posted November 16, 2021. The copyright holder for this preprint (which was not certified by peer review) is the author/funder, who has granted medRxiv a license to display the preprint in perpetuity.

It is made available under a CC-BY-NC 4.0 International license .

A

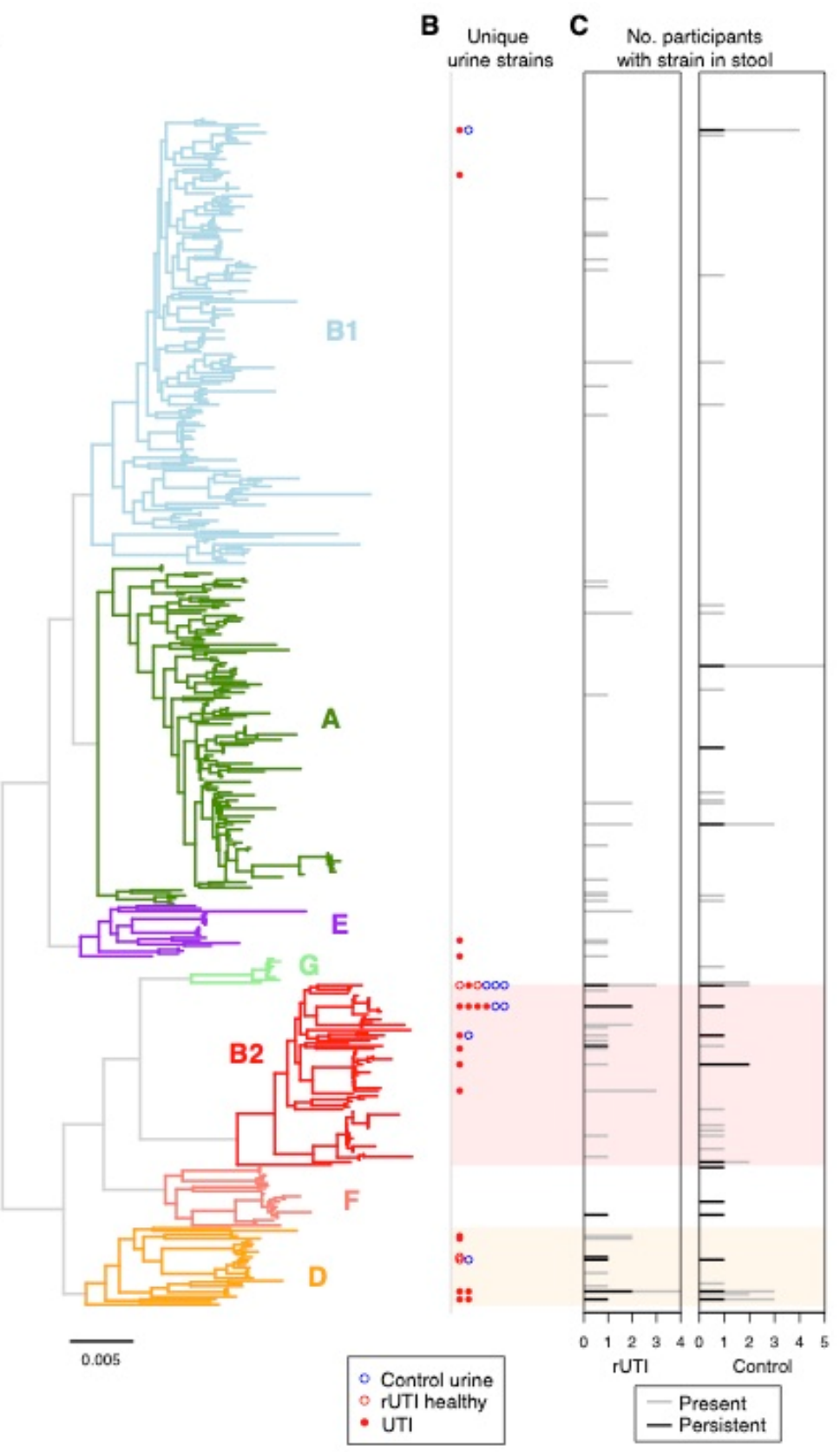

Figure 4. Phylogenetic distribution of $E$. coli strains identified in all stool and urine samples. (a) The phylogenetic tree of StrainGE reference strains colored and annotated by phylogroup. (b) Unique E. coli strains identified in urine samples are marked alongside the corresponding reference strain. Filled circles represent UTI-causing strains, blue circles denote strains identified in control hosts. (c) The total number of rUTI (left) and control (right) women with the corresponding strain present in stool samples. Black bars denote the number of women for whom the strain was persistent in the gut. 
medRxiv preprint doi: https://doi.org/10.1101/2021.11.15.21266268; this version posted November 16, 2021. The copyright holder for this preprint (which was not certified by peer review) is the author/funder, who has granted medRxiv a license to display the preprint in perpetuity.

It is made available under a CC-BY-NC 4.0 International license .

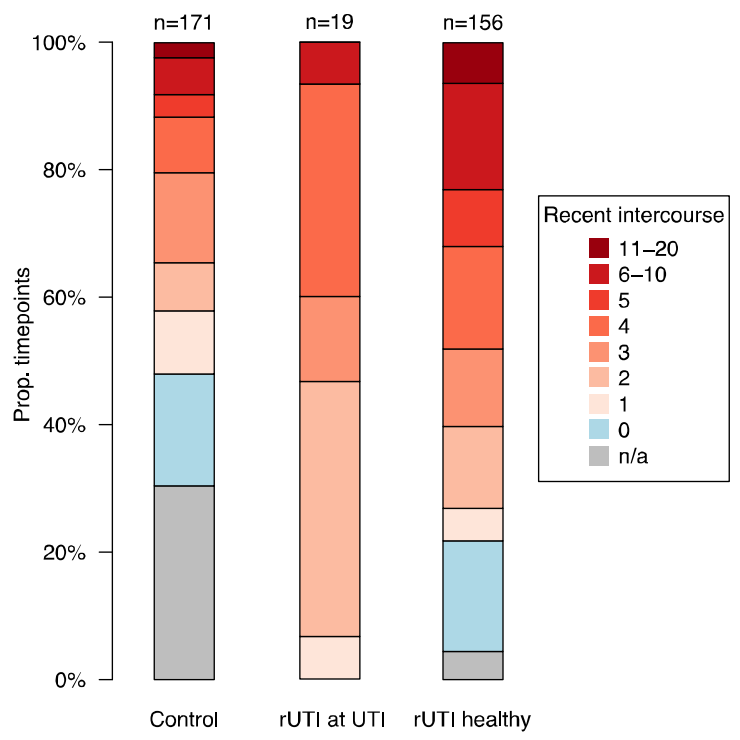

Figure S1. Sex precedes all clinical UTI events. Survey reports of intercourse frequency in the previous two weeks. Responses are partitioned by (i) control women, (ii) rUTI women at time of UTI, and (iii) rUTI women at non-UTI time points.

A
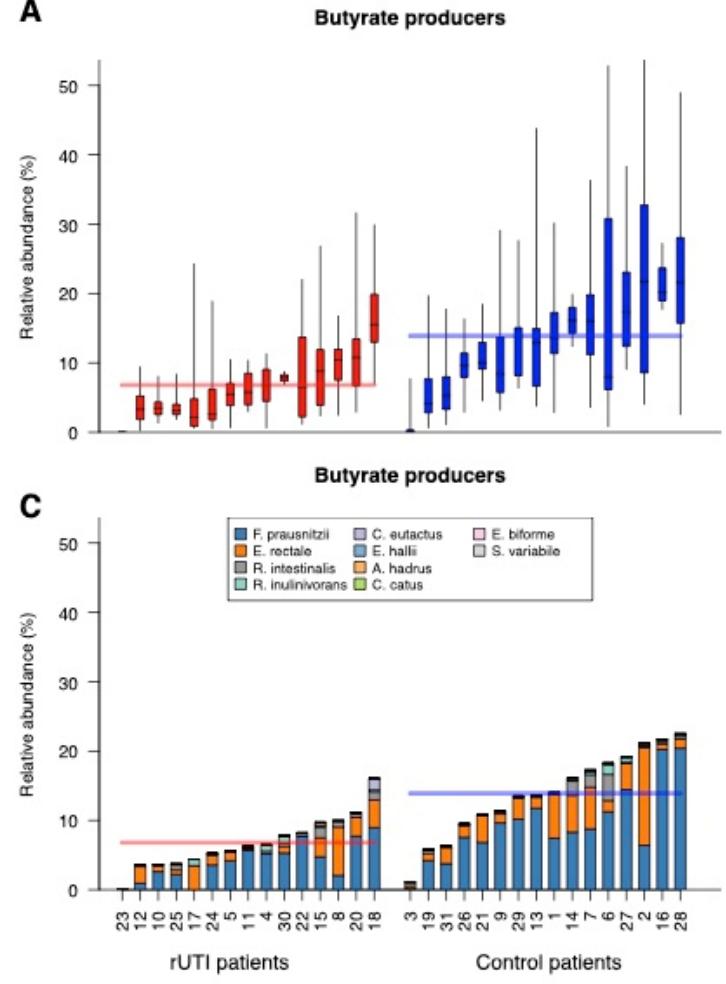

B

Propionate producers

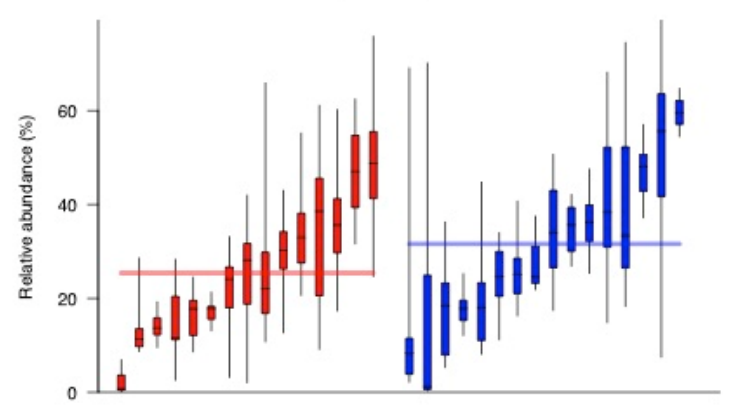

D

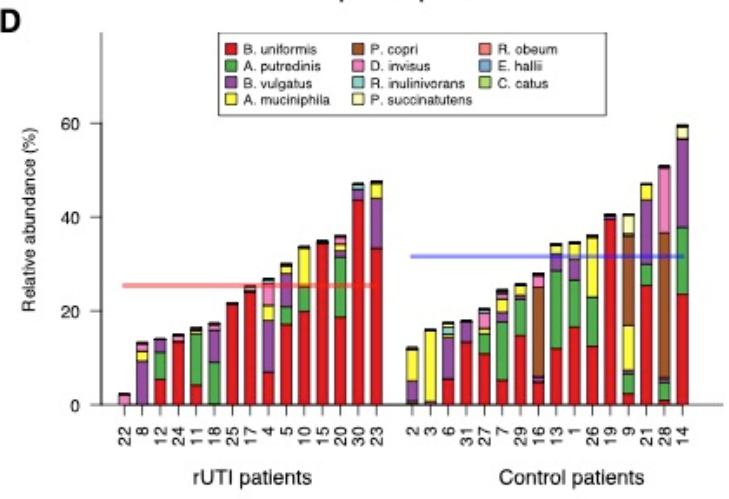

Figure S2. SCFA producing bacteria are depleted in the rUTI gut. Cumulative relative abundances of (a) butyrate and (b) propionate producing bacterial species in rUTI and control samples. Box plots denote the IQR and 95\% central quantile. Within-host average relative 
abundances of individual species for (c) butyrate and (d) propionate producers are also shown.
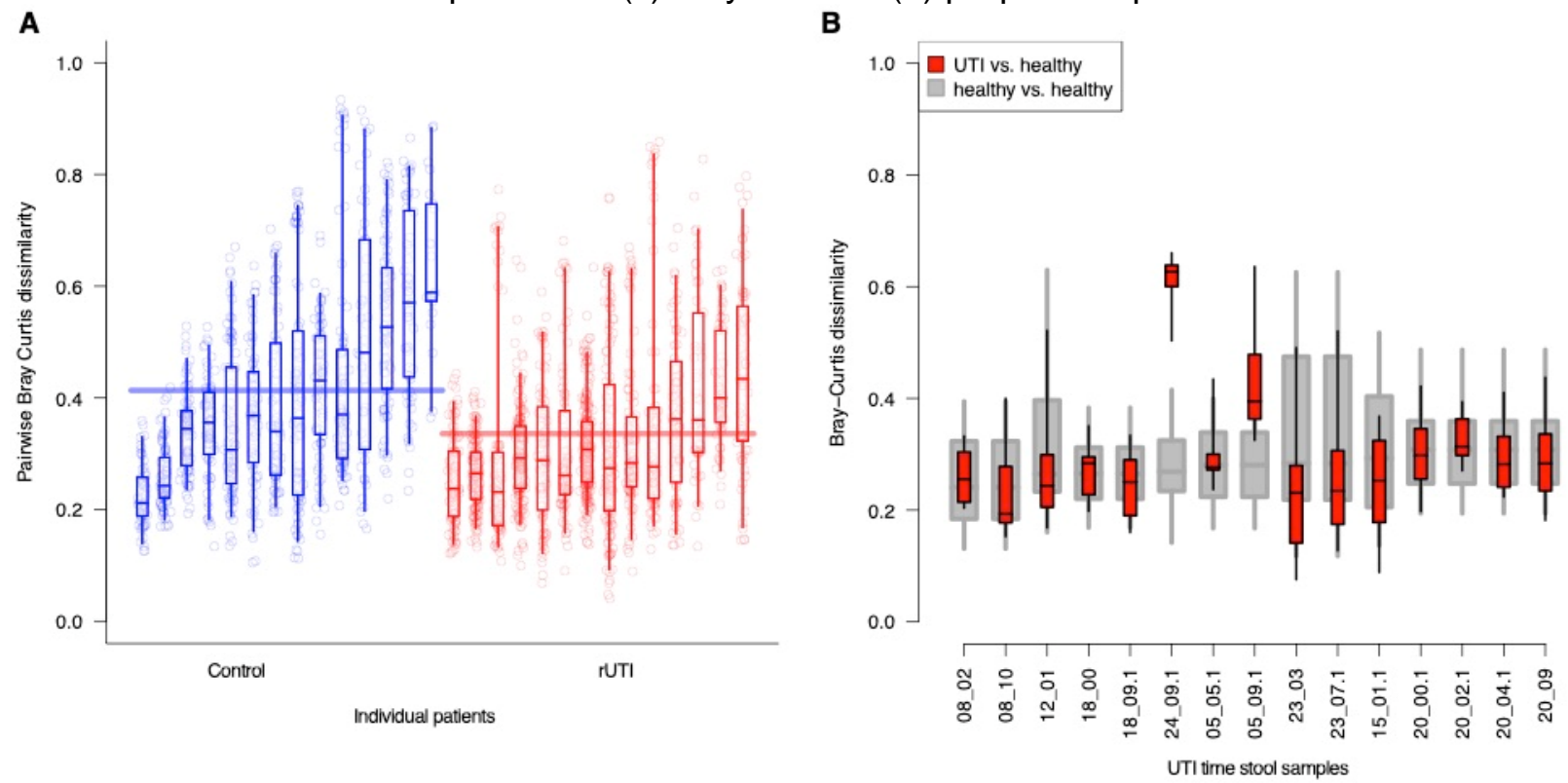

Figure S3. Bray Curtis dissimilarity across stool samples. (a) For each patient, the distribution of Bray-Curtis dissimilarities between all stool samples, ordered by increasing mean patient values within each cohort. (b) Bray-Curtis distributions between samples taken at the time of UTI vs. healthy time points (red), compared to all pairwise healthy sample comparisons. Box plots denote IQR \& 95\% central quantile 
medRxiv preprint doi: https://doi.org/10.1101/2021.11.15.21266268; this version posted November 16, 2021. The copyright holder for this preprint (which was not certified by peer review) is the author/funder, who has granted medRxiv a license to display the preprint in perpetuity.

It is made available under a CC-BY-NC 4.0 International license .
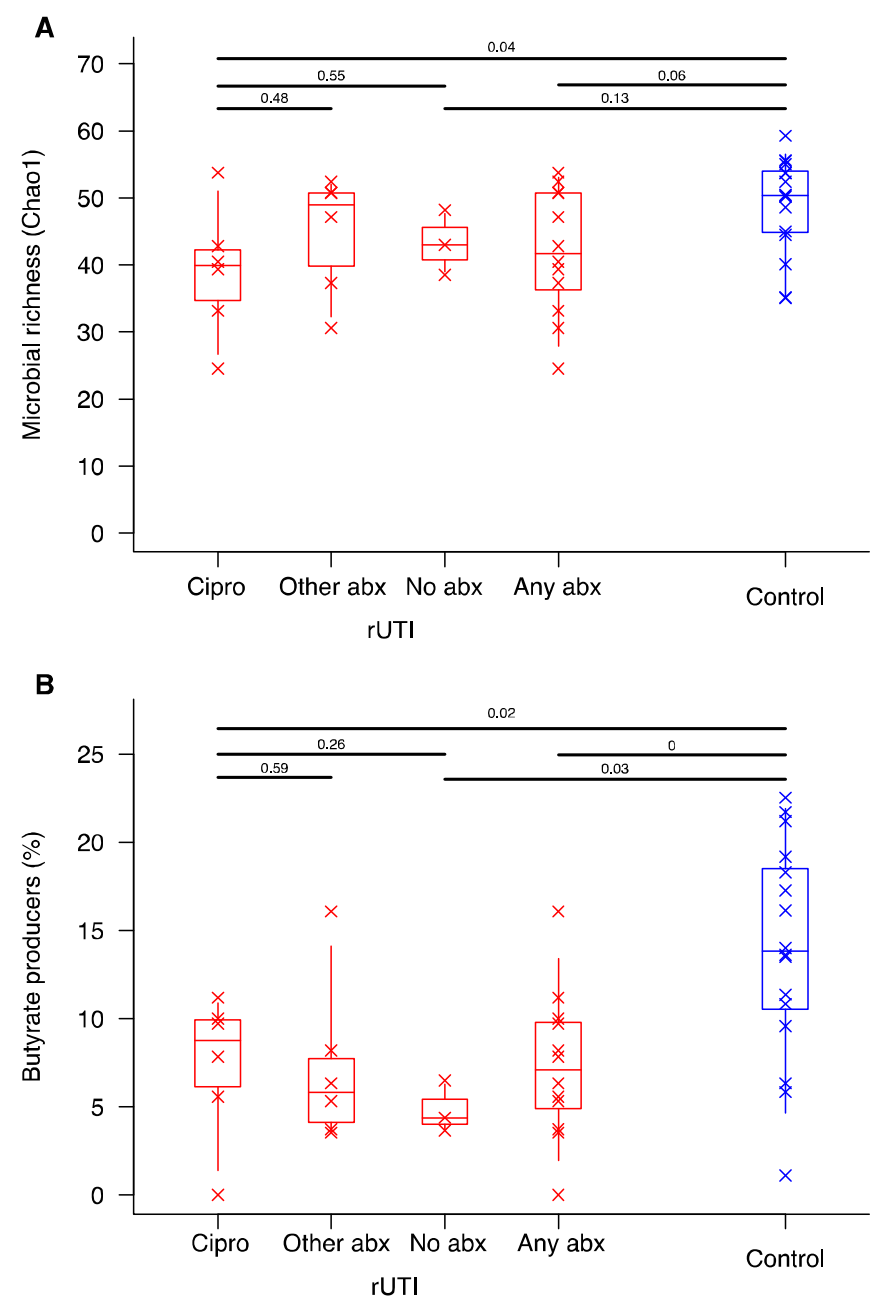

Figure S4. rUTI dysbiosis is not driven by antibiotic use during the study. We grouped rUTI women according to their antibiotic exposures at any point during the UMB study; (i) ciprofloxacin (ii) non-ciprofloxacin antibiotics; (iii) no antibiotics; (iv) any antibiotics. Groups were compared against each other and against the control cohort for (a) overall microbial richness and (b) relative abundance of butyrate producers. Crosses represent mean values for individuals, boxplots denote the IQR and 95\% central quantiles for each group. Wilcoxon rank sum tests were applied to group pairs to derive p-values. 


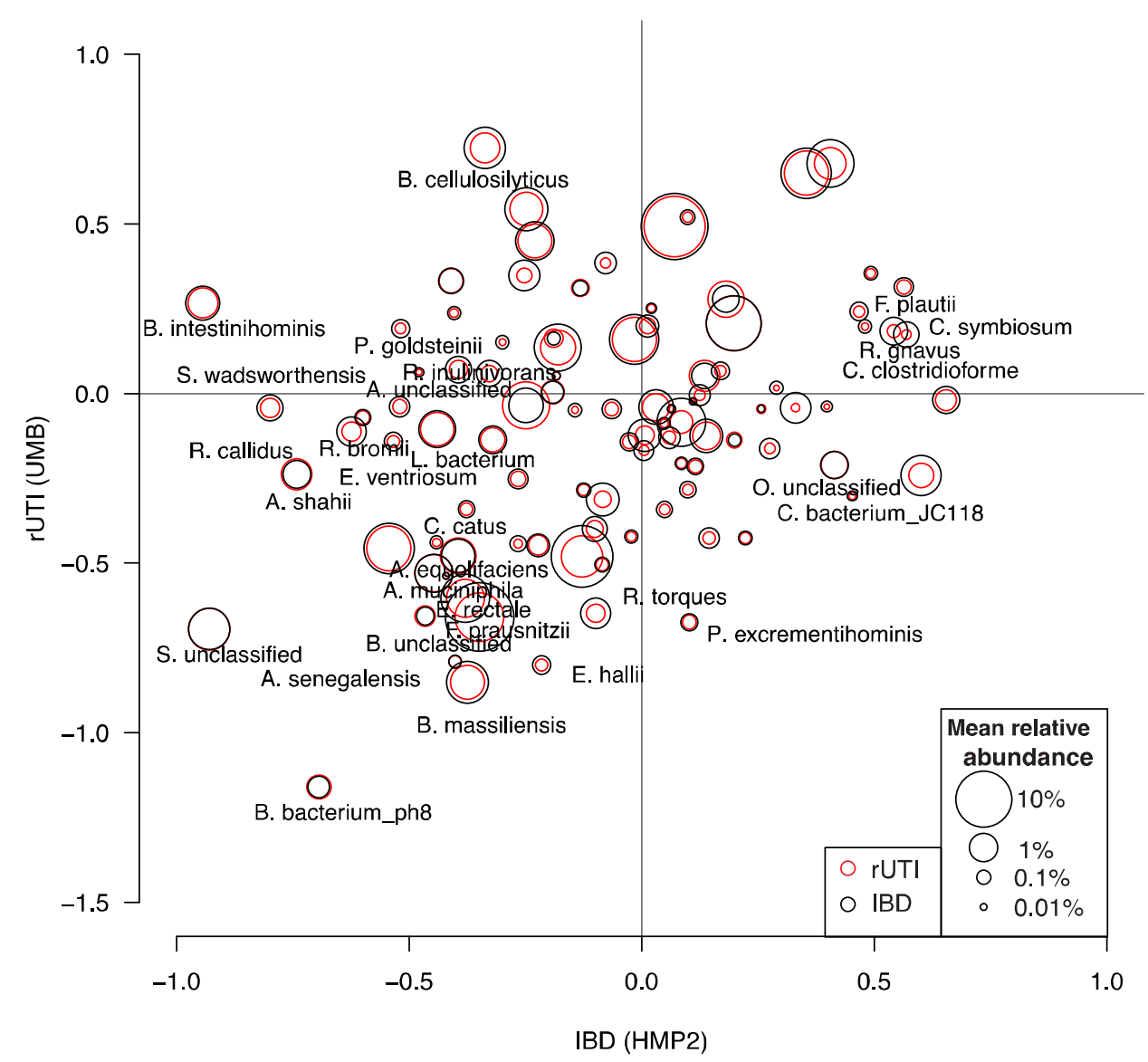

Figure S5. Most species depleted in the rUTI gut are also depleted in the IBD gut. We compared discriminatory taxa in rUTI women to those in IBD patients using data from adult participants in the HMP2 study ${ }^{33}$. For each study, we fitted mixed effects models to standardized Metaphlan2 relative abundances as a function of categorical disease group (rUTI or IBD respectively, vs. each study's control cohort), including covariates for race and antibiotic use. The disease group coefficients are plotted against each other for each species, with circle pairs representing the average relative abundance in each study. Species with uncorrected $p$ values $<0.05$ in either study are labelled. Species not present in at least $10 \%$ of samples in either study are excluded. IBD comprises patients with either CD or UC. 
a

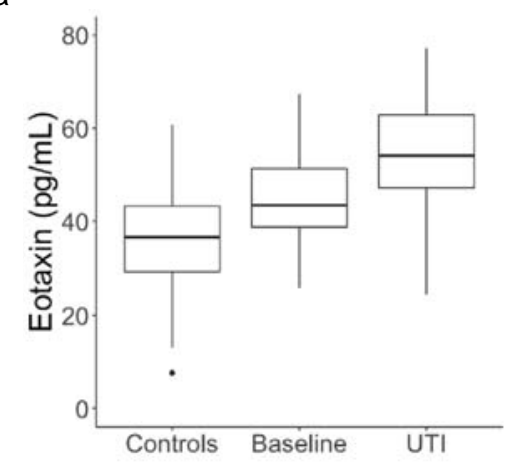

b

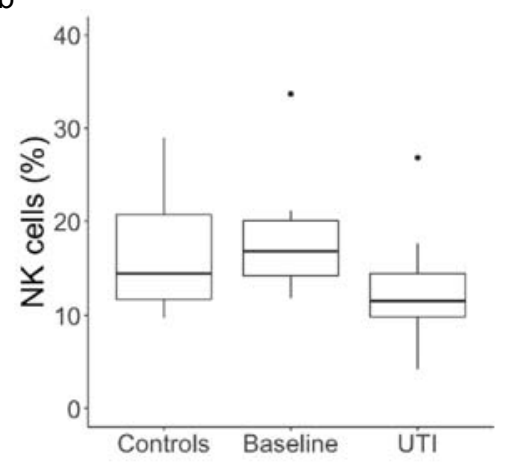

Figure S6. Immunological differences between cohorts. (a) Plasma eotaxin-1 levels in control women, and rUTI women at healthy enrollment and time of UTI. (b) Relative abundance of NK cells in control and rUTI women based on CIBERSORT output.

A

vs. median relative abundance

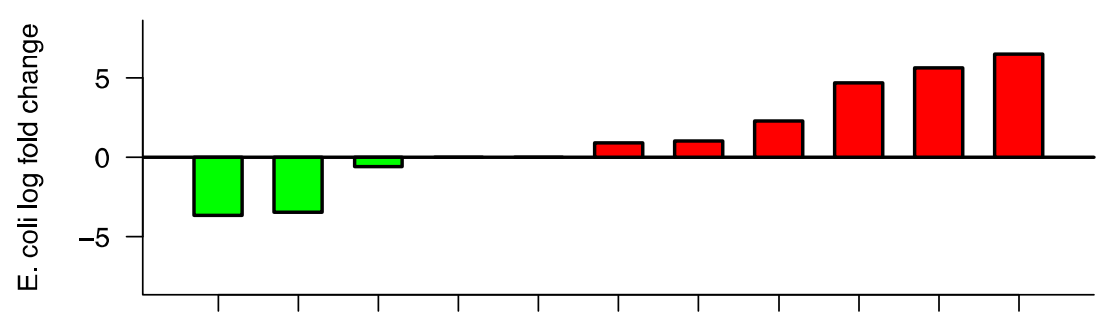

B

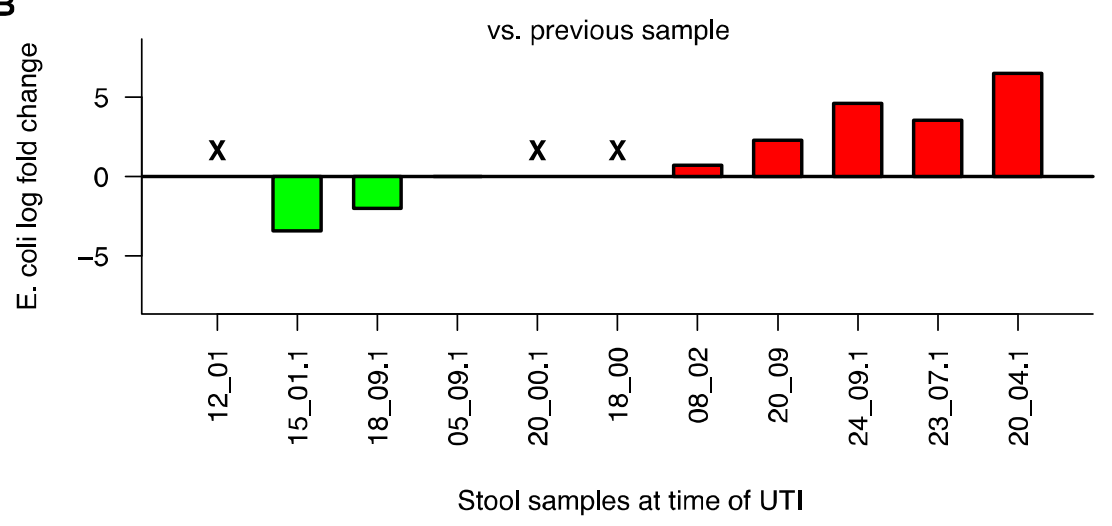

Figure S7. E. coli relative abundance around the time of UTI. For all stool samples taken within 3 days of a UTI event, the log fold change is given relative to (a) the median E. coli relative abundance in the corresponding patient, excluding samples taken at the time of UTI, and (b) the relative abundance of $E$. coli in the preceding stool sample. ' $X$ ' denotes samples for which there was no prior sample available. 
medRxiv preprint doi: https://doi.org/10.1101/2021.11.15.21266268; this version posted November 16, 2021. The copyright holder for this preprint (which was not certified by peer review) is the author/funder, who has granted medRxiv a license to display the preprint in perpetuity.

It is made available under a CC-BY-NC 4.0 International license.

A

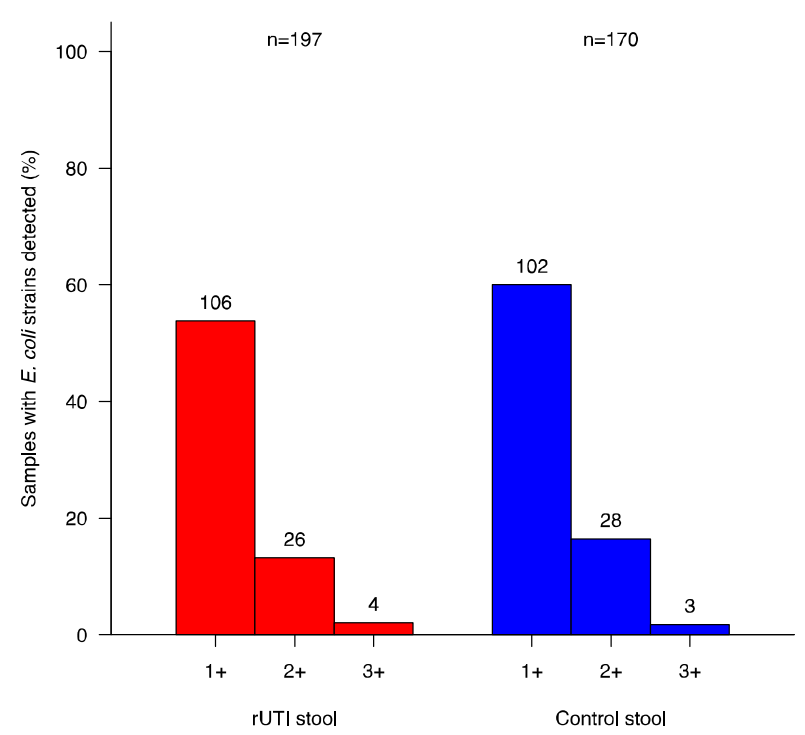

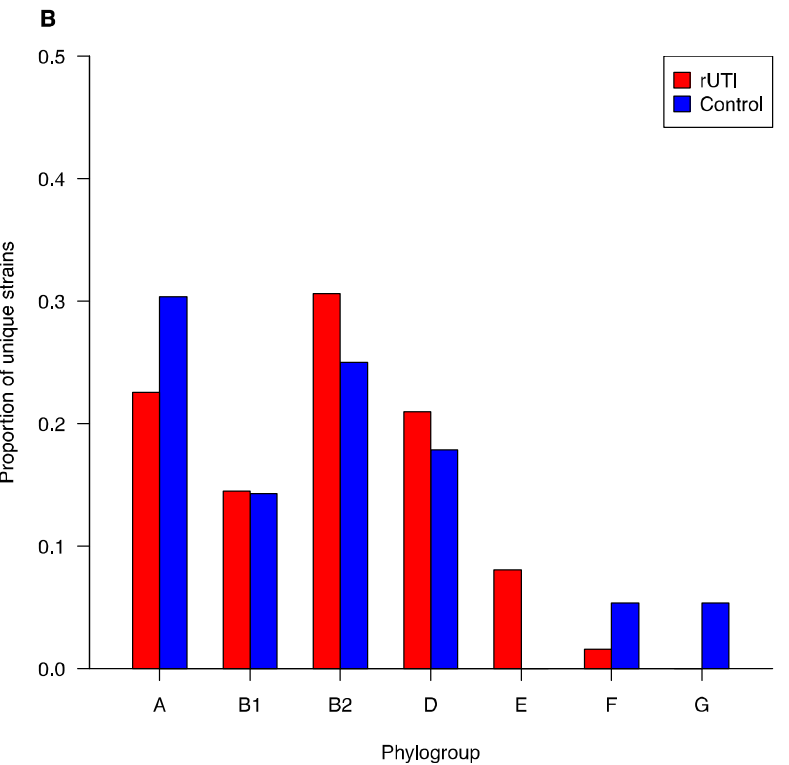

Figure S8. E. coli strain detection with StrainGE. (a) Number of detected E. coli strains by sample type. (b) Number of detected StrainGST reference strains vs. relative abundance of $E$. coli. 
medRxiv preprint doi: https://doi.org/10.1101/2021.11.15.21266268; this version posted November 16, 2021. The copyright holder for this preprint (which was not certified by peer review) is the author/funder, who has granted medRxiv a license to display the preprint in perpetuity.

It is made available under a CC-BY-NC 4.0 International license.

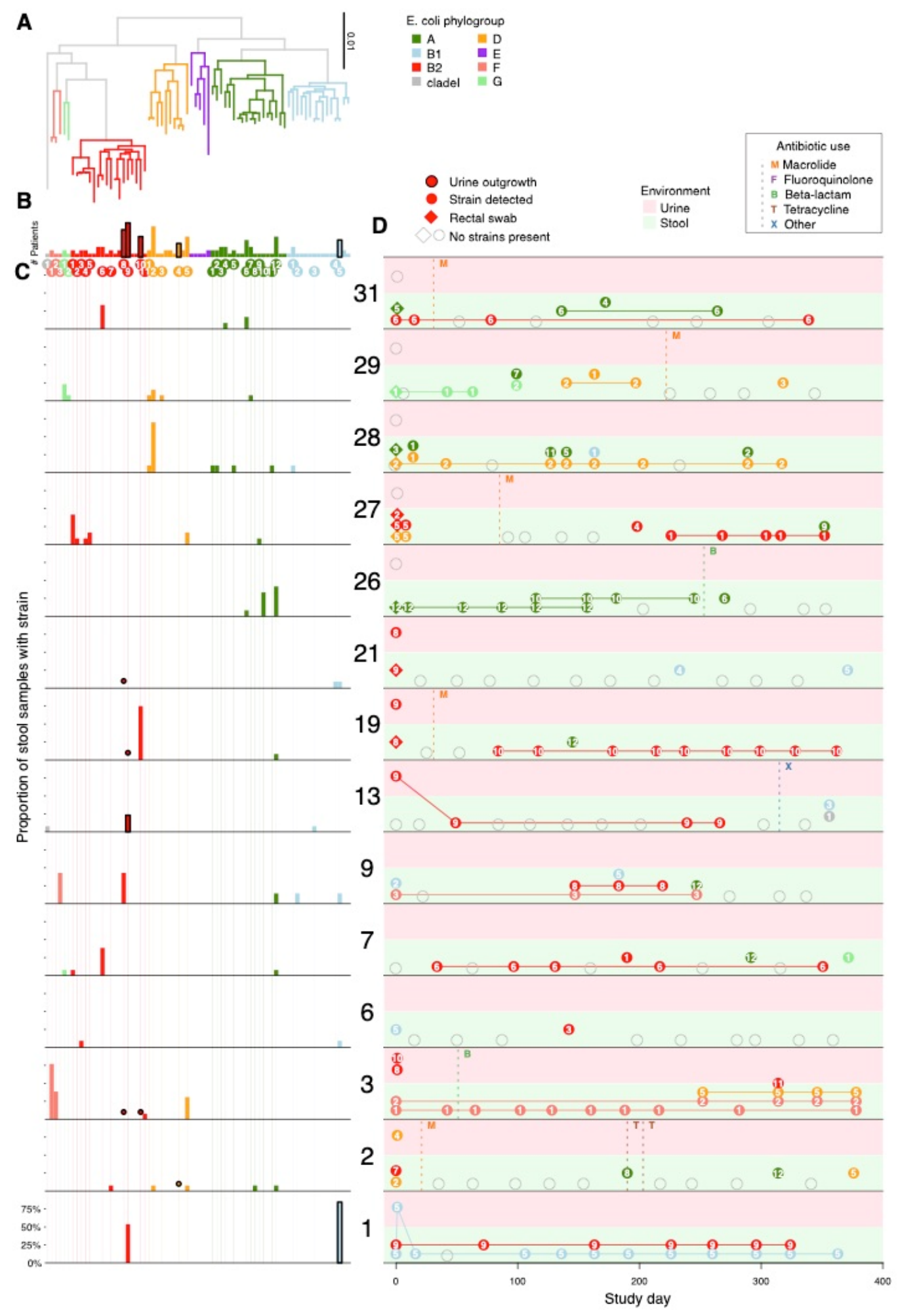

Figure S9. Strain dynamics in control women. Analogous to Figure 3 for the control cohort. 


\section{Supplementary Tables}

Table S1. Cohort characteristics. Demographic, behavioral and dietary characteristics of the rUTI and control women who completed the year-long study. Fisher's exact test used to compare frequencies between cohorts.

Table S2. Summary of UTls and urine sequence data in the UMB study. For each diagnosed UTIs, sequence data were available for cultured (plated on MacConkey agar) and/or uncultured urine samples. Metaphlan2 was used to estimate taxonomic composition and E. coli relative abundance. UTI species was determined to be the most abundant species associated with urovirulence. StrainGST provided representative $E$. coli reference strain(s), for which the corresponding clades and sequence types (STs) are also provided. The day of UTI is provided relative to the patient's enrollment sample. Inferred UTIs did not have corresponding urine samples.

Table S3. Differential taxa between rUTI and control cohorts. At the species, genus and family levels, all bacterial taxa which differed between rUTI and control women with FDR<0.25. The fitted rUTI coefficient is estimated in a multivariable mixed effects regression model, adjusting for recent antibiotic use and race. Multiple testing correction applied separately at each taxonomic level.

Table S4. Differential KEGG Orthogroups between rUTI and control cohorts. Abundances of KEGG Orthogroups, derived with HUMAnN2, were compared across cohorts using linear mixed models. The fitted rUTI coefficient is estimated in a multivariable mixed effects regression model, adjusting for recent antibiotic use and race. All hits with FDR $<0.05$ are provided. KEGG Orthogroups belonging to butyrate production pathways ${ }^{62}$ are marked.

Table S5. Differential pathways between rUTI and control cohorts. Abundances of pathways derived with HUMAnN2, were compared across cohorts using linear mixed models. The fitted rUTI coefficient is estimated in a multivariable mixed effects regression model, adjusting for recent antibiotic use and race.

Table S6. Differential expression in PBMC RNA-Seq data. Using DESeq, we sought differentially expressed genes between the rUTI and control cohorts. All genes with FDR<0.5 are listed.

Table S7. E. coli in enrollment urine samples. All urine samples collected at enrollment in the absence of UTI symptoms are described. The dominant species was determined by MetaPhlan2, and the relative abundance of $E$. coli strains provided by StrainGE.

Table S8. Virulence factor enrichment in urine samples and $E$. coli database. Virulence factors enriched in UTI urine samples vs. StrainGE E. coli database. Frequency across the 15 urine samples is provided alongside frequency in the database, partitioned by clades B2/D vs. 
non-B2/D. Fisher's exact tests were performed to compare counts in urine vs. the $E$. coli database, as well as clade B2 vs. clade D within the database.

Table S9. Outgrowths from stool samples post-antibiotic exposures. A collection of stool samples for which $E$. coli strains appeared to have dropped out post antibiotic exposure were plated on selective media to determine if $E$. coli remained present at levels below detection limits for sequence data.

Table S10. Antibiotic resistance across strains associated with bladder colonization. $E$. coli strains were isolated from rectal swabs collected at enrollment and UTI diagnosis timepoints and submitted to antibiotic susceptibility profiling. Phenotypic resistance was compared to resistance gene content. Resistance breakpoints were: Nitrofurantoin $\geq 128 \mathrm{ug} / \mathrm{ml}$; Ciprofloxacin $\geq 0.12 \mathrm{ug} / \mathrm{ml}$; Amoxicillin $\geq 32 \mathrm{ug} / \mathrm{ml}$; Sulfamethoxazole-Trimethoprim $\geq 16 \mathrm{ug} / \mathrm{ml}$; azithromycin $\geq 32 \mathrm{ug} / \mathrm{ml}$ (https://www.fda.gov/media/108180/download). Resistance determinants were derived using Resistance Gene Identifier ${ }^{63}$. 


\section{References}

1. Flores-Mireles, A.L., Walker, J.N., Caparon, M. \& Hultgren, S.J. Urinary tract infections: epidemiology, mechanisms of infection and treatment options. Nat Rev Microbiol 13, 269-284 (2015).

2. Hooton, T.M., et al. A prospective study of risk factors for symptomatic urinary tract infection in young women. N Engl J Med 335, 468-474 (1996).

3. Yamamoto, S., et al. Genetic evidence supporting the fecal-perineal-urethral hypothesis in cystitis caused by Escherichia coli. J Urol 157, 1127-1129 (1997).

4. Nielsen, K.L., Dynesen, P., Larsen, P. \& Frimodt-Moller, N. Faecal Escherichia coli from patients with E. coli urinary tract infection and healthy controls who have never had a urinary tract infection. J Med Microbio/ 63, 582-589 (2014).

5. Jantunen, M.E., Saxen, H., Lukinmaa, S., Ala-Houhala, M. \& Siitonen, A. Genomic identity of pyelonephritogenic Escherichia coli isolated from blood, urine and faeces of children with urosepsis. J Med Microbiol 50, 650-652 (2001).

6. Magruder, M., et al. Gut uropathogen abundance is a risk factor for development of bacteriuria and urinary tract infection. Nat Commun 10, 5521 (2019).

7. Thanert, R., et al. Comparative Genomics of Antibiotic-Resistant Uropathogens Implicates Three Routes for Recurrence of Urinary Tract Infections. mBio 10(2019).

8. Paalanne, N., et al. Intestinal microbiome as a risk factor for urinary tract infections in children. Eur J Clin Microbiol Infect Dis 37, 1881-1891 (2018).

9. Magruder, M., et al. Gut commensal microbiota and decreased risk for Enterobacteriaceae bacteriuria and urinary tract infection. Gut Microbes 12, 1805281 (2020).

10. Tariq, R., et al. Fecal Microbiota Transplantation for Recurrent Clostridium difficile Infection Reduces Recurrent Urinary Tract Infection Frequency. Clin Infect Dis 65, 17451747 (2017).

11. Wang, T., Kraft, C.S., Woodworth, M.H., Dhere, T. \& Eaton, M.E. Fecal Microbiota Transplant for Refractory Clostridium difficile Infection Interrupts 25-Year History of Recurrent Urinary Tract Infections. Open Forum Infect Dis 5, ofy016 (2018).

12. Mayer, E.A., Tillisch, K. \& Gupta, A. Gut/brain axis and the microbiota. J Clin Invest 125, 926-938 (2015).

13. Cryan, J.F., et al. The Microbiota-Gut-Brain Axis. Physiol Rev 99, 1877-2013 (2019).

14. Budden, K.F., et al. Emerging pathogenic links between microbiota and the gut-lung axis. Nat Rev Microbiol 15, 55-63 (2017).

15. Dang, A.T. \& Marsland, B.J. Microbes, metabolites, and the gut-lung axis. Mucosal Immunol 12, 843-850 (2019).

16. Lazar, V., et al. Aspects of Gut Microbiota and Immune System Interactions in Infectious Diseases, Immunopathology, and Cancer. Front Immunol 9, 1830 (2018).

17. Scholes, D., et al. Risk factors for recurrent urinary tract infection in young women. $J$ Infect Dis 182, 1177-1182 (2000).

18. Clemente, J.C., Manasson, J. \& Scher, J.U. The role of the gut microbiome in systemic inflammatory disease. BMJ 360, j5145 (2018).

19. Belkaid, Y. \& Hand, T.W. Role of the microbiota in immunity and inflammation. Cell 157, 121-141 (2014).

20. Parada Venegas, D., et al. Short Chain Fatty Acids (SCFAs)-Mediated Gut Epithelial and Immune Regulation and Its Relevance for Inflammatory Bowel Diseases. Front Immunol 10, 277 (2019).

21. Liu, H., et al. Butyrate: A Double-Edged Sword for Health? Adv Nutr 9, 21-29 (2018). 
22. Kanehisa, M., Sato, Y., Kawashima, M., Furumichi, M. \& Tanabe, M. KEGG as a reference resource for gene and protein annotation. Nucleic Acids Res 44, D457-462 (2016).

23. Franzosa, E.A., et al. Species-level functional profiling of metagenomes and metatranscriptomes. Nat Methods 15, 962-968 (2018).

24. Mack, A., et al. Changes in gut microbial metagenomic pathways associated with clinical outcomes after the elimination of malabsorbed sugars in an IBS cohort. Gut Microbes 11, 620-631 (2020).

25. Palleja, A., et al. Recovery of gut microbiota of healthy adults following antibiotic exposure. Nat Microbiol 3, 1255-1265 (2018).

26. Zaura, E., et al. Same Exposure but Two Radically Different Responses to Antibiotics: Resilience of the Salivary Microbiome versus Long-Term Microbial Shifts in Feces. mBio 6, e01693-01615 (2015).

27. Rooney, A.M., et al. Each Additional Day of Antibiotics Is Associated With Lower Gut Anaerobes in Neonatal Intensive Care Unit Patients. Clin Infect Dis 70, 2553-2560 (2020).

28. Schubert, A.M., et al. Microbiome data distinguish patients with Clostridium difficile infection and non-C. difficile-associated diarrhea from healthy controls. mBio 5, e0102101014 (2014).

29. Pozuelo, M., et al. Reduction of butyrate- and methane-producing microorganisms in patients with Irritable Bowel Syndrome. Sci Rep 5, 12693 (2015).

30. Geirnaert, A., et al. Butyrate-producing bacteria supplemented in vitro to Crohn's disease patient microbiota increased butyrate production and enhanced intestinal epithelial barrier integrity. Sci Rep 7, 11450 (2017).

31. Ni, J., Wu, G.D., Albenberg, L. \& Tomov, V.T. Gut microbiota and IBD: causation or correlation? Nat Rev Gastroenterol Hepatol 14, 573-584 (2017).

32. Schaubeck, M., et al. Dysbiotic gut microbiota causes transmissible Crohn's disease-like ileitis independent of failure in antimicrobial defence. Gut 65, 225-237 (2016).

33. Integrative Human Microbiome Project Research Network Consortium. The Integrative Human Microbiome Project: dynamic analysis of microbiome-host omics profiles during periods of human health and disease. Cell Host Microbe 16, 276-289 (2014).

34. Zhou, Y. \& Zhi, F. Lower Level of Bacteroides in the Gut Microbiota Is Associated with Inflammatory Bowel Disease: A Meta-Analysis. Biomed Res Int 2016, 5828959 (2016).

35. Duvallet, C., Gibbons, S.M., Gurry, T., Irizarry, R.A. \& Alm, E.J. Meta-analysis of gut microbiome studies identifies disease-specific and shared responses. Nat Commun $\mathbf{8}$, 1784 (2017).

36. Asnicar, F., et al. Blue poo: impact of gut transit time on the gut microbiome using a novel marker. Gut (2021).

37. Takahashi, D., et al. Microbiota-derived butyrate limits the autoimmune response by promoting the differentiation of follicular regulatory T cells. EBioMedicine 58, 102913 (2020).

38. Rosser, E.C., et al. Microbiota-Derived Metabolites Suppress Arthritis by Amplifying ArylHydrocarbon Receptor Activation in Regulatory B Cells. Cell Metab 31, 837-851 e810 (2020).

39. Li, F., Wang, M., Wang, J., Li, R. \& Zhang, Y. Alterations to the Gut Microbiota and Their Correlation With Inflammatory Factors in Chronic Kidney Disease. Front Cell Infect Microbiol 9, 206 (2019).

40. Adar, T., Shteingart, S., Ben Ya'acov, A., Bar-Gil Shitrit, A. \& Goldin, E. From airway inflammation to inflammatory bowel disease: eotaxin-1, a key regulator of intestinal inflammation. Clin Immunol 153, 199-208 (2014). 
41. Adar, T., et al. The Importance of Intestinal Eotaxin-1 in Inflammatory Bowel Disease: New Insights and Possible Therapeutic Implications. Dig Dis Sci 61, 1915-1924 (2016).

42. Cheung, W., Bluth, M., Khan, S., Johns, C. \& Bluth, M. Peripheral blood mononuclear cell gene array profiles in female patients with involuntary bladder contractions. Advances in Genomics and Genetics 1, 3-7 (2011).

43. de Santiago, P.R., et al. Immune-related IncRNA LINC00944 responds to variations in ADAR1 levels and it is associated with breast cancer prognosis. Life Sci 268, 118956 (2021).

44. Gur, C., et al. Natural killer cell-mediated host defense against uropathogenic E. coli is counteracted by bacterial hemolysinA-dependent killing of NK cells. Cell Host Microbe 14, 664-674 (2013).

45. Rivera-Chavez, F., et al. Depletion of Butyrate-Producing Clostridia from the Gut Microbiota Drives an Aerobic Luminal Expansion of Salmonella. Cell Host Microbe 19, 443-454 (2016).

46. Antharam, V.C., et al. Intestinal dysbiosis and depletion of butyrogenic bacteria in Clostridium difficile infection and nosocomial diarrhea. J Clin Microbiol 51, 2884-2892 (2013).

47. Clermont, O., Bonacorsi, S. \& Bingen, E. Rapid and simple determination of the Escherichia coli phylogenetic group. Appl Environ Microbiol 66, 4555-4558 (2000).

48. Schreiber, H.L.t., et al. Bacterial virulence phenotypes of Escherichia coli and host susceptibility determine risk for urinary tract infections. Sci Trans/ Med 9(2017).

49. van Dijk, L., et al. StrainGE: A toolkit to track and characterize low-abundance strains in complex microbial communities. bioRxiv 2021.02.14.431013(2021).

50. Garretto, A., et al. Genomic Survey of E. coli From the Bladders of Women With and Without Lower Urinary Tract Symptoms. Front Microbiol 11, 2094 (2020).

51. Zhang, S., et al. Short Chain Fatty Acids Modulate the Growth and Virulence of Pathosymbiont Escherichia coli and Host Response. Antibiotics (Basel) 9(2020).

52. Stapleton, A.E. The Vaginal Microbiota and Urinary Tract Infection. Microbiol Spectr 4(2016).

53. Cusumano, C.K., et al. Treatment and prevention of urinary tract infection with orally active FimH inhibitors. Sci Trans/ Med 3, 109ra115 (2011).

54. Truong, D.T., et al. MetaPhIAn2 for enhanced metagenomic taxonomic profiling. Nat Methods 12, 902-903 (2015).

55. Dobin, A., et al. STAR: ultrafast universal RNA-seq aligner. Bioinformatics 29, 15-21 (2013).

56. Liao, Y., Smyth, G.K. \& Shi, W. featureCounts: an efficient general purpose program for assigning sequence reads to genomic features. Bioinformatics 30, 923-930 (2014).

57. Robinson, M.D., McCarthy, D.J. \& Smyth, G.K. edgeR: a Bioconductor package for differential expression analysis of digital gene expression data. Bioinformatics 26, 139140 (2010).

58. Newman, A.M., et al. Robust enumeration of cell subsets from tissue expression profiles. Nat Methods 12, 453-457 (2015).

59. Love, M.I., Huber, W. \& Anders, S. Moderated estimation of fold change and dispersion for RNA-seq data with DESeq2. Genome Biol 15, 550 (2014).

60. Bankevich, A., et al. SPAdes: a new genome assembly algorithm and its applications to single-cell sequencing. J Comput Biol 19, 455-477 (2012).

61. Louis, P. \& Flint, H.J. Formation of propionate and butyrate by the human colonic microbiota. Environ Microbiol 19, 29-41 (2017).

62. Levi, I., et al. Potential role of indolelactate and butyrate in multiple sclerosis revealed by integrated microbiome-metabolome analysis. Cell Rep Med 2, 100246 (2021). 
medRxiv preprint doi: https://doi.org/10.1101/2021.11.15.21266268; this version posted November 16, 2021. The copyright holder for this preprint (which was not certified by peer review) is the author/funder, who has granted medRxiv a license to display the preprint in perpetuity.

It is made available under a CC-BY-NC 4.0 International license.

63. Alcock, B.P., et al. CARD 2020: antibiotic resistome surveillance with the comprehensive antibiotic resistance database. Nucleic Acids Res 48, D517-D525 (2020). 